\title{
Optical spectroscopy of type 2 LINERs
}

\author{
L. Hermosa Muñoz, S. Cazzoli, I. Márquez, and J. Masegosa
}

Instituto de Astrofísica de Andalucía - CSIC, Glorieta de la Astronomía s/n, 18008 Granada, Spain

e-mail: lhermosa@iaa.es

Received 12 September 2019 / Accepted 31 December 2019

\begin{abstract}
Context. Type 2 Low-ionization Nuclear Emission-line Regions (LINERs) have been optically classified with the Palomar data as not presenting a broad component in the Balmer emission lines that are associated with the broad-line region (BLR) of the active galactic nuclei (AGN).

Aims. We aim to unveil the presence of different kinematic components of emission lines in the nuclear region of a sample of local $(z \leq 0.022)$ type 2 LINERs. We focus on the analysis of the true nature of LINERs by means of the detection (or nondetection) of a broad component that originated in the BLR of the AGN. Additionally, we search for the possible presence of nonrotational motions such as outflows in these LINERs.

Methods. We applied a decomposition of the nuclear emission lines using an spectroscopic analysis of the optical spectra of nine type 2 LINERs of intermediate-resolution spectroscopic data retrieved from the Hubble Space Telescope (HST) archive. The study is completed with archival spectra from the Double Spectrograph from the Palomar Observatory.

Results. The emission line fitting reveals the presence of a broad component associated with the BLR in six out of the nine galaxies for the space-based data, and for two out of the eight from the ground-based spectra. The velocity dispersion for two galaxies (NGC 4486 and NGC 4594) measured in HST/STIS data suggest the presence of outflows.

Conclusions. The results indicate that the spatial resolution plays a major role in the detection of the BLR, as it appears diluted in the ground-based data (even after removing stellar contribution). This is also true for the emission line diagnostics, as the contaminant light contributes to lower emission line ratios toward the star-forming area of standard BPTs. We propose to reclassify NGC 4594 as a type 1 LINER, since a BLR component is seen in both space- and ground-based spectra. We find ambiguous results for the BLR component of NGC 4486. The modest outflow detection in our sample may indicate that they are not as frequent as seen for type 1 LINERs.
\end{abstract}

Key words. galaxies: active - galaxies: nuclei - galaxies: kinematics and dynamics

\section{Introduction}

Low-ionization Nuclear Emission-line Regions (LINERs) are among the lowest luminosity active galactic nuclei (AGN) found in the Universe. Defined for the first time by Heckman (1980), their spectra is dominated by low-ionization lines in contrast with the importance of high-ionization emission lines ([O III] $\lambda 5007$ or He II $\lambda 4686$ ) in more luminous AGNs, such as Seyferts. The initial separation between LINERs and Seyferts was defined by Heckman (1980) based on the relative ratios between the intensity of several emission lines ([O II] $] 33727$ to [O III] $\lambda 5007$ and [O I] $\lambda 6300$ to [O III] $\lambda 5007)$.

LINERs may be the most numerous type of AGNs in the local Universe (Ho et al. 1997), although their true AGN nature is still a matter of debate (Ho 2008; Márquez et al. 2017). Their spectral features cannot be explained only with stellar formation processes (Heckman 1980), and an AGN is not the only valid explanation. Other mechanisms that could reproduce their spectral features are, for example, shock ionization (Heckman 1980; Dopita \& Sutherland 1995; Dopita et al. 1996) and photoionization by post-AGB stars (Binette et al. 1994; Stasińska et al. 2008; Papaderos et al. 2013).

The morphology of the circumnuclear $\mathrm{H} \alpha$ emission as seen by the HST imaging indicates the possible presence of outflowing material in some of the systems (Pogge et al. 2000; Masegosa et al. 2011). In these cases, the outflow could contribute to the broadening of emission lines such as $\mathrm{H} \alpha-[\mathrm{N} \mathrm{II}]$, [O I], and [S II]. This could compromise the detection of the weak emission of Balmer lines from the broad-line region (BLR) in their nucleus. Shocks associated with outflows may also be important in ionizing the nuclear gas (and surroundings) (Dopita et al. 1996; Molina et al. 2018). However, these are observed at lower velocities (Molina et al. 2018) than those needed by the shock models (Groves et al. 2004) to reproduce LINER-like line ratios.

As Seyfert galaxies, LINERs appear as type 1.9 or type 2, depending on if the BLR is detected on their nucleus. The BLR is identified as a very broad component in the profiles of the Balmer lines. There are only a few systematic works studying the AGN nature of LINERs (Ho et al. 2003; Cazzoli et al. 2018), with most of them being individual discoveries (Bower et al. 1996; Ho et al. 1997).

In the work by Cazzoli et al. (2018), the spectra for a sample of nearby $(z<0.025)$ type 1.9 LINERs obtained with both ground- and space-based telescopes are analyzed. A broad component indicative of the BLR is required for the fitting of groundbased data in only a third of the targets, despite the fact that they were all classified as type 1.9 by Ho et al. (1997). Nevertheless, the modeling of the spectra of the targets obtained with the Space Telescope Imaging Spectrograph (STIS), mounted on the Hubble Space Telescope (HST), requires a broad $\mathrm{H} \alpha$ feature.

As with type 2 LINERs the BLR is not detected, the AGN nature of these systems is still controversial. Other components, 
such as post-AGB stars or low-mass X-ray binaries may play an important role in explaining their optical spectra (see Ho et al. 1997; McKernan et al. 2010).

In this paper, we analyze HST spectra of nine type 2 LINERs selected from the sample of González-Martín et al. (2009) with available data in the archive to investigate if they present a broad component in the Balmer lines that could be associated with the existence of a BLR in their nuclei. We completed the study with a comparison to the ground-based spectra from Ho et al. (1995), since the majority of the targets were classified as type 2 objects on that work. We also revisited the possible influence of the outflows in the broadening of Balmer lines, following Cazzoli et al. (2018).

This paper is organized as follows: in Sect. 2 we describe the sample and the data reduction process. Section 3 indicates the spectral line fitting for each nuclear spectra. Sections 4 and 5 show the main modeling results for space- and ground-based spectra, respectively. In Sect. 6, we discuss the modeling results, the possible presence of a BLR in some of the targets, and a comparison of the two datasets for both kinematics and line ratios. Finally, in Sect. 7, we present a summary and the main conclusions of the work.

\section{Sample and data processing}

The sample selection is drawn from the survey by GonzálezMartín et al. (2009) of 82 LINERs selected at X-rays. From this sample, we selected all type 2 LINERs classified as AGNs from the analysis of their features in the wavelength range from $\mathrm{X}$-rays to the near-infrared. The final sample of 12 galaxies comprises all the objects with available spectra in the HST/STIS archive with the intermediate resolution grating G750M $(R \sim$ 5000).

This grating covers a total wavelength range from 5450 to $10140 \AA$, centered at 6581 or $6768 \AA$. This range has been chosen due to the availability of the [S II] $\lambda \lambda 6716,6731 \AA$; [N II] $] \lambda 6548,6584 \AA$; and $\mathrm{H} \alpha \lambda 6563 \AA$ lines in all the spectra and [O I] $\lambda \lambda 6300,6364 \AA$ in those where the grating was centered at $6581 \AA$ (all except NGC 4594 and NGC 4676B). Two out of 12 were discarded because the low signal-to-noise ratio $(\mathrm{S} / \mathrm{N})$ of the spectra (NGC 4261 and NGC 5055, for which Constantin et al. 2015 already reported the poor quality of the nuclear spectrum of the latter), and also NGC 6240, because even though its spectrum is archived as nuclear, the emission lines were not visible. This leaves a total of nine galaxies in the final sample of type 2 LINERs. Basic information is provided in Table 1.

The full calibrated 2D spectra were retrieved from the HST archive. In Table 2, the main observing details of each nuclear spectrum analyzed are listed. Then, we extracted the 1D flux and wavelength-calibrated nuclear spectra of the sources. Some of them (namely NGC 4698, NGC 4552, NGC 4676B) showed abundant cosmic rays (CR). The results could be improved by performing a new CR correction with the L.A. CosMIC algorithm (van Dokkum 2001) to the flat-fielded frames also downloaded from the archive. After this correction, we applied the last step of the reduction process following the HST pipeline, which is the $\mathrm{X} 2 \mathrm{D}$ task (under the STSDAS package in IRAF ${ }^{1}$ ). This task generates the final calibrated frame and corrects the geometrical distortion.

For the galaxies with several nuclear exposures available, they were realigned and combined with the IRAF tasks IMSHIFT

\footnotetext{
1 IRAF is the Image Reduction and Analysis Facility distributed by the National Optical Astronomy Observatories (NOAO) for the reduction and analysis of astronomical data. http://iraf.noao.edu/
}

and IMCOMBINE. These tasks improved the $\mathrm{S} / \mathrm{N}$ after the $\mathrm{CR}$ rejection. If there was only one nuclear spectrum available, and the CRs fell close to the lines in the majority of the nuclear rows, we extracted each row individually and did the CR removal manually. Then, the individual rows were combined to form the final nuclear spectra. If a CR fell on the continuum of these spectra, it was masked in the analysis.

The final nuclear spectrum was extracted using APALL task in IRAF. The number of extracted and combined rows depends on the slit width and the binning of the CCD. The majority of the targets were measured with a slit width of $52^{\prime \prime} \times 0.2^{\prime \prime}$, a plate scale of $0.05^{\prime \prime} \mathrm{pixel}^{-1}$, and no binning. For these, the five central rows (pixels) were extracted to form the final spectra. For the spectra binned to $0.1^{\prime \prime}$ pixel $^{-1}$, the three central rows were extracted. All the galaxies observed with a slit width of $52^{\prime \prime} \times 0.1^{\prime \prime}$ were unbinned, and therefore we extracted five rows. The number of pixels extracted for each galaxy and its equivalent spatial scale are indicated in Table 2 (Col. 6).

However, the data for two of the objects (NGC 4552 and NGC 4676B) had low S/N that did not improve with the combination of multiple exposures. For NGC 4552, there were additional spectra from regions near the very center, so a line-fitting was possible for the nuclear spectrum (see Sect. 3). We also fit the NGC 4676B spectrum, but it was finally excluded due to the low significance of the obtained modeling, as is discussed in Sect. 6.

Additionally, we searched for the spectra of the nine objects within the sample by Ho et al. (1995) in order to compare the space-based and ground-based spectra. The latter were retrieved from the archive. They were observed with the Double Spectrograph (Oke \& Gunn 1982), located in the Cassegrain focus of the Hale $5 \mathrm{~m}$ telescope at Palomar Observatory during 1984 and 1985. The long-slit spectra were measured with a 1200 line $\mathrm{mm}^{-1}$ grating blazed to cover a wavelength range $\sim 6210-6860 \AA$. The spectral resolution was $R \sim 2500$ for a $2^{\prime \prime}-$ length slit and $R \sim 4000$ for a $1^{\prime \prime}$-length slit. Spectra for eight out of the nine galaxies (all except NGC 4676B, member of a merging system, which is located at approximately ten times the distance of the rest of the targets, see Table 1) were retrieved already reduced from the NASA/IPAC Extragalactic Database $(\mathrm{NED})^{2}$ archive. The width of the slit was $2^{\prime \prime} \times 128^{\prime \prime}$ for all the targets, except for NGC 4486, which was $1^{\prime \prime} \times 128^{\prime \prime}$. The total extraction window was $2^{\prime \prime} \times 4^{\prime \prime}$ (for NGC 4486 was $1^{\prime \prime} \times 4^{\prime \prime}$ ), equivalent to summing up the central seven pixels of the CCD. The mean seeing of the observations is $1.5^{\prime \prime}$. The spectral and spatial resolutions were $\sim 2.5 \AA$ and $0.58^{\prime \prime} \mathrm{pixel}^{-1}$, respectively.

\section{Analysis of the nuclear spectra}

The procedure of the spectral analysis is done as explained below.

\subsection{Stellar subtraction}

The host galaxy contribution on the nuclear spectra of a lowluminosity AGN may be significant, thus the starlight should be subtracted to robustly measure the kinematics and fluxes of the emission lines. However, due to the small wavelength range covered for the HST/STIS data ( $572 \AA$ ), we decided not to perform the subtraction as the line-free continuum regions of the spectra are small for a proper stellar continuum modeling. Moreover, Constantin et al. (2015; hereafter C15) proved that the correction

\footnotetext{
2 The NASA/IPAC Extragalactic Database (NED) is funded by the National Aeronautics and Space Administration and operated by the California Institute of Technology.
} 
Table 1. General properties for nine type 2 LINERs discussed in this paper.

\begin{tabular}{|c|c|c|c|c|c|c|c|c|}
\hline ID & $\begin{array}{c}\text { RA } \\
\text { (hh:mm:ss) } \\
(2)\end{array}$ & $\begin{array}{c}\text { Dec } \\
(\mathrm{dd}: \mathrm{mm}: \mathrm{ss}) \\
(3)\end{array}$ & $\begin{array}{c}\text { Morphology } \\
\text { (4) }\end{array}$ & (5) & $\begin{array}{c}\text { Scale } \\
\left.(\mathrm{pc} \mathrm{arcsec})^{-1}\right) \\
(6)\end{array}$ & $\begin{array}{c}i \\
\left({ }^{\circ}\right) \\
(7)\end{array}$ & $\begin{array}{c}V_{\text {rot }} \\
\left(\mathrm{km} \mathrm{s}^{-1}\right) \\
(8)\end{array}$ & $\begin{array}{l}\text { PA } \\
\left({ }^{\circ}\right) \\
(9)\end{array}$ \\
\hline NGC 2685 & 085534.71 & +584403.83 & $(\mathrm{R}) \mathrm{SB} 0+\mathrm{pec}^{(a)}$ & 0.0029 & 70 & 60 & $146 \pm 4$ & 38 \\
\hline NGC 3245 & 102718.387 & +283026.79 & $\mathrm{SA}(\mathrm{r}) 0^{(b)}$ & 0.0045 & 90 & 58 & - & 177 \\
\hline NGC 4374 & 122503.74 & +125313.14 & $\mathrm{E} 1^{(c)}$ & 0.0034 & 65 & - & $189 \pm 15$ & 135 \\
\hline NGC 4486 & 123049.42 & 122328.04 & $\mathrm{E} 0+\operatorname{pec}^{(d)}$ & 0.0043 & 84 & - & - & 160 \\
\hline NGC 4552 & 123539.81 & +123322.83 & $\mathrm{E}^{(a)}$ & 0.0011 & 17 & - & - & - \\
\hline NGC 4594 & 123959.43 & -113722.99 & $\mathrm{SA}(\mathrm{s}) \mathrm{a}^{(a)}$ & 0.0034 & 59 & 68 & $408 \pm 11$ & 90 \\
\hline NGC 4676B & 124611.24 & +304321.87 & $\mathrm{SB}(\mathrm{s}) 0 / \mathrm{a}+\mathrm{pec}^{(a)}$ & 0.0220 & 472 & - & $397 \pm 31$ & - \\
\hline NGC 4698 & 124822.91 & +082914.58 & $\mathrm{SA}(\mathrm{s}) \mathrm{ab}^{(a)}$ & 0.0034 & 64 & 53 & 518 & $203 \pm 3$ \\
\hline NGC 4736 & 125053.061 & +410713.65 & $(\mathrm{R}) \mathrm{SA}(\mathrm{r}) \mathrm{ab}^{(a)}$ & 0.0010 & 25 & 36 & $182 \pm 5$ & 105 \\
\hline
\end{tabular}

Notes. (2) RA and (3) Dec: coordinates of the galaxy; (4) morphology: Hubble classification; (5) z and (6) scale: heliocentric redshifts and scale distance from the Local Group from NED; (7) $i$ : inclination angle from Ho et al. (1997); (8) $V_{\text {rot }}$ and (9) PA: maximum rotation velocity corrected from inclination and position angle from HyperLeda, respectively.

References. ${ }^{(a)}$ Ho et al. (1997); ${ }^{(b)}$ Wardle \& Knapp (1986); ${ }^{(c)}$ Huchtmeier (1994); ${ }^{(d)}$ Huchtmeier \& Richter (1986).

Table 2. Observing log of HST/STIS data.

\begin{tabular}{|c|c|c|c|c|c|c|c|c|}
\hline ID & $\begin{array}{c}\text { Other name } \\
\text { (2) }\end{array}$ & $\begin{array}{c}\text { Filename } \\
\left(\_\mathrm{sx} 2 / \_\mathrm{x} 2 \mathrm{~d}\right) \\
(3)\end{array}$ & $\begin{array}{c}\text { Proposal } \\
\text { ID } \\
(4)\end{array}$ & $\begin{array}{l}\text { PA } \\
\left({ }^{\circ}\right) \\
(5) \\
\end{array}$ & $\begin{array}{l}\text { Extr. window } \\
\text { pix }(\mathrm{pc}) \\
(6)\end{array}$ & $\begin{array}{c}\text { Obs. date } \\
(\mathrm{yy}-\mathrm{mm}-\mathrm{dd}) \\
(7)\end{array}$ & $\begin{array}{l}\text { Exp. time } \\
\text { (s) } \\
(8)\end{array}$ & $\begin{array}{c}\text { Im. filter } \\
(9)\end{array}$ \\
\hline NGC $2685^{(*)}$ & IRAS $08517+5855$ & o63n01020 & 8607 & 54 & $3(21)$ & $2001-05-06$ & 3097 & F814W \\
\hline NGC 3245 & IRAS $10245+2845$ & o57205030 & 7403 & 203 & $5(23)$ & 1999-02-02 & 2715 & F547M \\
\hline NGC 4374 & M 84 & o3wn01010/2010 & 7124 & 104 & $5(17)$ & 1997-04-14 & $1993 / 2223$ & F814W \\
\hline NGC 4486 & M 87 & o67z01010/2010 & 8666 & 164 & $5(21)$ & $2001-03-25 / 27$ & 1360 & F606W \\
\hline NGC 4552 & M 89 & 051203020 & 8472 & 78 & $5(4)$ & 2000-06-19 & 1440 & F555W \\
\hline NGC 4594 & M 104 & o4d303030 & 7354 & 250 & $5(15)$ & 1999-02-05 & 280 & F814W \\
\hline NGC 4676B ${ }^{(*)}$ & IC 820 & o67q09030 & 8669 & 195 & $3(144)$ & $2002-02-20$ & 740 & F814W \\
\hline NGC 4698 & IRAS $12458+0845$ & $\mathrm{o} 4 \mathrm{e} 022010 / 20 / 30$ & 7361 & 259 & $5(16)$ & $1997-11-24$ & $900 / 933 / 840$ & F606W \\
\hline NGC 4736 & M94 & o67110030 & 8591 & 50 & $5(6)$ & $2002-07-15$ & 1440 & F555W \\
\hline
\end{tabular}

Notes. Columns indicate: (1) galaxy name; (2) other name; (3) file name of the analyzed spectra as indicated in the archive; (4) proposal ID of the observations; (5) position angle of the slit (north-eastward); (6) extraction window and its corresponding scale in parsecs; (7) date of the observation; (8) exposure time of the spectra; (9) filter used for the sharp-divided image (Márquez \& Moles 1996) obtained also from the HST archive. The latter are shown in Appendix B. ${ }^{(*)}$ Spectrum obtained with a slit width whose plate scale was $0.102^{\prime \prime}$ pixel $^{-1}$. It is $0.051^{\prime \prime}$ pixel ${ }^{-1}$ for the rest of the objects.

is negligible for HST/STIS spectra in this wavelength range due to the small aperture of the instrument, which reduces the contaminant starlight from the host galaxy.

The starlight was modeled and subtracted for the Palomar data using a penalized PiXel fitting analysis (PPXF version 4.71 Cappellari \& Emsellem 2004; Cappellari 2017). This differs to the stellar modeling applied by Ho et al. (1993), in which template galaxies were used instead (see Sect. 5.2). The methodology of the stellar subtraction was done following a similar technique as in Cazzoli et al. (2018; hereafter C18), similarly to the modeling of the spectral emission lines, which is explained in below. For each object, the restframe velocity used in the analysis is defined as: $c \times z$ (Col. 5, Table 1). The starlight decontamination for the Palomar data and all the line modelings to the different spectra are found in Appendices A and B, respectively.

\subsection{Emission line fitting}

Each of the emission lines present in the spectra ([S II], [N II], $\mathrm{H} \alpha$ and in some cases [O I], Table 3, Col. 4) were fit with Gaussian functions to derive their properties: central wavelength, line width $(\sigma)$ and full width half maximum (FWHM), flux, and $f_{\text {blend }}$, defined as the percentage of the broad component flux with respect to the total flux of the $\mathrm{H} \alpha-[\mathrm{N}$ II] lines. The fitting was performed using a nonlinear least-squares minimization and curve-fitting routine (LMFIT) implemented in PYTHON, which is an extension of the Levenberg-Marquardt method found in the SCIPY package.

The modeling of the lines was performed with three different methods using either [S II], [O I], or both as templates for the $\mathrm{H} \alpha-[\mathrm{N}$ II] complex (Cazzoli et al. 2018, and references therein). As $\mathrm{H} \alpha$ and [N II] lines are generally blended, we do not use them directly, because the overall fit could be affected. Among those emission lines available, the best lines are the [O I] lines, as they are separated enough between them to serve as good templates. However, these lines are not always available or have low $\mathrm{S} / \mathrm{N}$ (e.g., see Fig. B.5), so we have to rely on [S II] lines like in previous works (Ho et al. 1997; Balmaverde \& Capetti 2014, Constantin et al. 2015). Therefore, the three different models we can apply are [S II], [O I], or both (if available) as the reference lines, because they are usually not strongly blended as $\mathrm{H} \alpha-[\mathrm{N} \mathrm{II}]$. 
Following Cazzoli et al. (2018), the first method is known as S-method, and it consists of fitting the [S II] lines and then tie to them the central wavelengths and widths of all the other narrow lines. The second method (O-method) is the same as $\mathrm{S}$-method, but it uses the [OI] lines as references. The third model is a mixed model (M-model), which uses [S II] and [O I] lines simultaneously as a template for the $\mathrm{H} \alpha-[\mathrm{N} \mathrm{II}]$ blend.

We tested different methods of fitting since the forbidden lines ions of the spectra have different critical densities ([S II] $10^{3} \mathrm{~cm}^{-3},[\mathrm{O} \mathrm{I}] \sim 10^{6} \mathrm{~cm}^{-3}$, and $[\mathrm{N} \mathrm{III}] \sim 10^{4} \mathrm{~cm}^{-3}$, respectively) caused by the possible stratification in the NLR. Therefore, the profiles of each line could be different (see Balmaverde \& Capetti 2014).

The fluxes of the two [N II] lines and the two [OI] lines were set to follow the relations $1: 3$ and 1:2.96, respectively (Osterbrock \& Ferland 2006). For more details on the methods and the constraints, we refer to C18.

As with $\mathrm{C} 18$, in order to prevent over fitting, we calculated a parameter, $\varepsilon_{\mathrm{c}}$, which is the standard deviation of a region of the continuum that did not have any absorption or emission line. This parameter was always calculated in a $50 \AA$-length region of the spectra. We compared it with the standard deviation of the residuals under each emission line, and if $\varepsilon^{\text {line }}<3 \times \varepsilon_{\mathrm{c}}$, then the fit is considered to be reliable. For the cases where $\varepsilon_{\mathrm{c}}$ was not sufficient in order to differentiate between two different fits, we used the $\chi^{2}$ of the fit and/or visual inspection as the last criteria to select the final model.

The procedure was organized in three steps. Firstly, we fit the continuum to a linear least squares model using the regions between the emission lines visible in the spectra.

Secondly, all lines were fit to a single Gaussian profile (hereinafter, "narrow component") with the same parameters for all of them. If the fit was satisfactory for the reference lines ([S II] or [O I] ), but not for [N II] and $\mathrm{H} \alpha$, then a broad Gaussian profile was added to $\mathrm{H} \alpha$ (hereinafter, "broad component"). However, if the fit was not reliable in any of the lines, or if the narrow component had a large velocity $\left(\geq 300 \mathrm{~km} \mathrm{~s}^{-1}\right)$, then a second component was added to all the lines (hereinafter, "secondary component").

Thirdly, if the $\mathrm{H} \alpha-[\mathrm{NII}]$ blend was not well-reproduced (significant residuals) with a narrow plus a secondary component, then we added a broad component in $\mathrm{H} \alpha$. All the line modelings are presented in the figures of Appendix B.

This method was not sufficient for the most complicated cases, for which additional restrictions were required. For example, the modeling of the reference lines for the NGC 4594 HST/STIS spectrum was good enough, but the maximum of the profiles of $[\mathrm{N} \mathrm{II}]$ and $\mathrm{H} \alpha$ seemed to be shifted $(\sim 1-3 \AA)$ from the maximum of the Gaussian profiles. Therefore, we shifted $1 \AA$ the maximum of the secondary Gaussian component to improve the fit. This produced a change in the velocity compatible within the initial error estimation.

For the HST/STIS spectra of two galaxies (namely NGC 4374 and NGC 4552), the [S II] lines were so blended, and the $[\mathrm{OI}]$ lines almost absent, that it was challenging to perform the modeling using them as reference (Figs. B.3 and B.5). Therefore, we decided to fit the spectrum of a region situated a few pixels away from the nucleus and use this result to model the narrow component of the nuclear region. This was motivated by the assumption that the narrow component is tracing the kinematics of the disk of the galaxy, thus it should also be visible in regions outside the very center of the system. Moreover, by moving to an outer part of the galaxy, the contribution of the
BLR (if present) should disappear due to its unresolved nature at HST scale. As the disk should be rotating with the same velocity dispersion, a modeling of this component in the spectra of an outer region would give approximately the same contribution we should expect in the nucleus, but with a shift in velocity. We note, though, that for some galaxies this condition is not completely true, as the disk velocity dispersion was found to increase towards the center (for NGC 3245 and NGC 4594 see Barth et al. 2001; Emsellem \& Ferruit 2000, respectively). This is discussed in more detail for each individual case in Sect. 6.3.2.

An additional assumption was made for NGC 4374. If the narrow component was fixed as it was with the previous method, when proceeding with the rest of the modeling, the secondary component was broad enough to be compatible with a very broad AGN component of $\sigma>800 \mathrm{~km} \mathrm{~s}^{-1}$. The addition of a third Gaussian in the $\mathrm{H} \alpha-[\mathrm{N} \mathrm{II}]$ complex instead generated a narrow profile to fit a peak that is visible in the spectrum (see Fig. B.3). Therefore, we exchanged the secondary and broad components to improve the fit.

We fixed the velocity dispersion of the narrow component for NGC 4374 and NGC 4552 with this method and allowed the velocity to vary in the nuclear spectra. This improved the final residuals (also visually) with respect to the normal fitting process.

The velocities $(V)$ and line widths $\left(\sigma_{\text {line }}\right)$ obtained for each galaxy, as well as the number of components and the model used to fit the reference lines, are indicated in Table 3. The S-method was used for all cases except for the Palomar spectrum of NGC 4552, for which we used the O-method. The line width has been corrected from the instrumental width by doing $\sigma_{\text {line }}=\sqrt{\sigma_{\text {obs }}^{2}-\sigma_{\text {inst }}^{2}}$, where the value of $\sigma_{\text {inst }}(\sim 1.34 \AA)$ has been taken from the HST/STIS handbook, and from Ho et al. (2009) for the Palomar data ( 2.2 $\AA$ ).

\section{Modeling of HST/STIS data}

After the summary of the overall modeling of the HST/STIS spectra, we compare the results with the work by Constantin et al. (2015). All the results from the spectra modeling are found in Appendix B.

\subsection{Overall modeling summary}

Despite there being three possible models to fit the lines, we selected the S-method to model all the HST/STIS spectra for different reasons. For five LINERs, the spectrum was lacking the [O I] lines either for the wavelength range coverage or because they were not detected (Table 3, Col. 4). For two LINERs, NGC 4374 and NGC 4552, the S/N of the [O I] lines was rather faint and difficult to model (see Sect. 3.2). For NGC 4736, the fit presented no significant difference when applying S-/O-/Mmodels, so we decided to use the simplest model that could explain the observed spectra, in this case, the S-model. For the remaining object, NGC 4486, although one of the [OI] lines is clearly visible (Fig. B.4), the modeling was better with the S-method (the $\chi^{2}$ improved about $\sim 30 \%$ ). All the fits to the different spectra can be found in the Appendix B.

Generally, the modeling of both [S II] and [O I] lines are inside the $3 \varepsilon_{\text {line }}$ limits for all LINERs except for NGC 4374 (see Fig. B.3). However, in the case of [N II] $-\mathrm{H} \alpha, \varepsilon_{\mathrm{c}}$ is approximately equal to $3 \varepsilon_{\text {line }}$ for NGC 4594 and $\sim 4 \varepsilon_{\text {line }}$ for NGC 4374 despite the addition of the very broad component (Fig B.6). In all the other objects, $\varepsilon_{\mathrm{c}}$ is within $3 \varepsilon_{\text {line }}$ limits, thus we consider the fit not to be well-constrained for these two cases. 
Table 3. Results from the analysis of the optical spectra.

\begin{tabular}{|c|c|c|c|c|c|c|c|c|c|c|}
\hline (1) & Inst. & Comp. & {$[\mathrm{OI}]$} & $\begin{array}{c}V_{\mathrm{N}}^{[\mathrm{S} \mathrm{II}]} \\
\left(\mathrm{km} \mathrm{s}^{-1}\right) \\
(5)\end{array}$ & $\begin{array}{c}\sigma_{\mathrm{N}}^{[\mathrm{S} \mathrm{II}]} \\
\left(\mathrm{km} \mathrm{s}^{-1}\right) \\
(6)\end{array}$ & $\begin{array}{c}V_{\mathrm{S}}^{[\mathrm{S} \mathrm{II}]} \\
\left(\mathrm{km} \mathrm{s}^{-1}\right) \\
(7)\end{array}$ & $\begin{array}{c}\sigma_{\mathrm{S}}^{[\mathrm{S} \mathrm{II}]} \\
\left(\mathrm{km} \mathrm{s}^{-1}\right) \\
(8)\end{array}$ & $\begin{array}{c}V_{\mathrm{B}}^{\mathrm{H} \alpha} \\
\left(\mathrm{km} \mathrm{s}^{-1}\right) \\
(9)\end{array}$ & $\begin{array}{c}\sigma_{\mathrm{B}}^{\mathrm{H} \alpha} \\
\left(\mathrm{km} \mathrm{s}^{-1}\right) \\
(10)\end{array}$ & $\begin{array}{c}f_{\text {blend }} \\
(\%) \\
(11)\end{array}$ \\
\hline \multirow[t]{2}{*}{ NGC 2685} & HST/STIS & $\mathrm{N}$ & $\mathrm{N}$ & $80 \pm 1$ & $63 \pm 12$ & - & - & - & - & - \\
\hline & Palomar & $\mathrm{N}$ & $Y$ & $-10 \pm 1$ & $70 \pm$ & - & - & - & - & - \\
\hline \multirow[t]{2}{*}{ NGC 3245} & HST/STIS & $\mathrm{N}+\mathrm{B}$ & $\mathrm{N}$ & $134 \pm 29$ & $172 \pm 6$ & - & - & $424 \pm$ & $998 \pm 6$ & 47 \\
\hline & Palon & $\mathrm{N}+\mathrm{S}$ & $\mathrm{Y}$ & $-216 \pm 9$ & $25 \pm 40$ & $94 \pm 17$ & $136 \pm 21$ & - & - & - \\
\hline \multirow[t]{2}{*}{ NGC 4374} & HST/STIS & $\mathrm{N}+\mathrm{S}+\mathrm{B}$ & $\mathrm{Y}$ & $-275 \pm 13$ & $146 \pm 14$ & $362 \pm 5$ & $281 \pm 24$ & $693 \pm 7$ & $1432 \pm 37$ & 65 \\
\hline & Palomar & $\mathrm{N}+\mathrm{S}$ & Y & $117 \pm 4$ & $51 \pm 29$ & $33 \pm 56$ & $395 \pm 71$ & - & - & - \\
\hline \multirow[t]{2}{*}{ NGC 4486} & HST/STIS & $\mathrm{N}+\mathrm{S}$ & Y & $395 \pm 4$ & $198 \pm 22$ & $-101 \pm 378$ & $655 \pm 18$ & - & - & - \\
\hline & Palomar $^{(*)}$ & $\mathrm{N}+\mathrm{S}+\mathrm{B}$ & Y & $-478 \pm 4$ & $238 \pm 5$ & $96 \pm 6$ & $286 \pm 9$ & $-285 \pm 145$ & $908 \pm 141$ & 5 \\
\hline \multirow[t]{2}{*}{ NGC $4552^{(\dagger),(\ddagger)}$} & HST/STIS & $\mathrm{N}+\mathrm{S}+\mathrm{B}$ & Y & $-103 \pm 47$ & $157 \pm 18$ & $501 \pm 38$ & $249 \pm 50$ & $541 \pm 53$ & $1360 \pm 20$ & 70 \\
\hline & Palomar ${ }^{(\dagger)}$ & $\mathrm{N}$ & Y & $56 \pm 59$ & $282 \pm 68$ & - & - & - & - & - \\
\hline \multirow[t]{2}{*}{ NGC 4594} & HST/STIS & $\mathrm{N}+\mathrm{S}+\mathrm{B}$ & $\mathrm{N}$ & $70 \pm 15$ & $108 \pm 26$ & $66 \pm 152$ & $554 \pm 6$ & $519 \pm$ & $1677 \pm$ & 16 \\
\hline & Palomar & $\mathrm{N}+\mathrm{B}$ & Y & $-11 \pm 4$ & $215 \pm 7$ & - & - & $698 \pm 26$ & $956 \pm 41$ & 33 \\
\hline NGC 4676B ${ }^{(*),(\ddagger)}$ & HST/STIS ${ }^{(\ddagger)}$ & $\mathrm{N}+\mathrm{B}$ & $\mathrm{N}$ & $527 \pm 40$ & $198 \pm 12$ & - & - & $336 \pm 16$ & $748 \pm 12$ & 89 \\
\hline \multirow[t]{2}{*}{ NGC 4698} & HST/STIS & $\mathrm{N}+\mathrm{B}$ & $\mathrm{N}$ & $55 \pm 19$ & $90 \pm 5$ & - & - & $273 \pm 97$ & $1375 \pm 9$ & 39 \\
\hline & Palomar & $\mathrm{N}$ & Y & $-7 \pm$ & $98 \pm 8$ & - & - & - & - & - \\
\hline \multirow[t]{2}{*}{ NGC 4736} & HST/STIS & $\mathrm{N}+\mathrm{B}$ & $\mathrm{Y}$ & $127 \pm 14$ & $84 \pm 12$ & - & - & $330 \pm 1$ & $738 \pm 4$ & 82 \\
\hline & Palomar & $\mathrm{N}$ & Y & $23 \pm 4$ & $105 \pm 10$ & - & - & - & - & - \\
\hline
\end{tabular}

Notes. From left to right: ID: object designation; Inst.: HST/STIS or Palomar spectra; Comp.: components used for the best-fitting model with $\mathrm{N}, \mathrm{S}$ and B referring to narrow, second, and broad components, respectively; [OI]: if these lines are present in the spectrum or not (Yes/No); velocity $(V)$ and velocity dispersion $(\sigma)$ for each component for each of the reference emission lines; $f_{\text {blend }}$ : contribution of the broad component to the total flux of the $\mathrm{H} \alpha-[\mathrm{NII}]$ complex. The galaxies in bold show a secondary component in the HST/STIS spectrum classified as outflow (see Sect. 6.2 and Fig. 2). ${ }^{(*)}$ Indicates that the broad component detection in $\mathrm{H} \alpha-[\mathrm{N} \mathrm{II}]$ lines is not well-constrained (Sect. 6.3). ${ }^{(\dagger)}$ The data for which the O-model was used to model the emission lines (see Sect. 3.2). ${ }^{(\$)}$ Marks the spectra with low S/N (Sect. 2).

We find that NGC 2685 is the only spectrum that can be modeled with a single narrow component in all the emission lines, and NGC 4486 is the only spectrum well-reproduced with a narrow plus a secondary component. No very broad component is required for them (Figs. B.1 and B.4). For the other seven LINERs, we found that four out of nine need one narrow Gaussian component to model all the forbidden lines, and an additional broad (BLR) component in $\mathrm{H} \alpha$ (see Table 3, Col. 3). On the other hand, the remaining three LINERs need a secondary component to model the forbidden lines and narrow $\mathrm{H} \alpha$ in addition to a very broad component in $\mathrm{H} \alpha$. In summary, a secondary component is needed in four out of the nine analyzed galaxies, and a very broad component in $\mathrm{H} \alpha$ is needed in seven out of the nine LINERs.

\subsection{Comparison with Constantin et al. (2015) (C15)}

Most of the galaxies shown here (7 out of 9; $77 \%$ of the sample) have already been analyzed by $\mathrm{C} 15$, where they report, for the first time, the detection of broad components in eight objects of their total sample. They have studied a total of 113 AGNs with HST/STIS and MMT (Multiple Mirror Telescope) spectroscopic data to analyze the emission lines and to give constraints on the ionizing mechanisms present in the galaxies. The reduction process of the HST/STIS data and the line modeling, for which they used the [S II] lines as template for the $\mathrm{H} \alpha-[\mathrm{N} \mathrm{II}]$ blend, follow a similar technique to ours. For the galaxies in common, they found a broad $\mathrm{H} \alpha$ emission for three of them (NGC 3245, NGC 4594, NGC 4736) in agreement with our findings (see Table 3 and Figs. B.2, B.6 and B.9).

A direct comparison of the contributions of the very broad component to the $\mathrm{H} \alpha-[\mathrm{N} \mathrm{II}]$ complex and the FWHM for these three galaxies can be seen in Fig. 1. Galaxy NGC 3245 is represented with an empty or filled symbol depending on the number of components used for the modeling. Figure 1 shows that, if we consider our two-component modeling, the contribution of the very broad component to the $\mathrm{H} \alpha-[\mathrm{N} \mathrm{II}]$ complex is compatible in both analyses. Nevertheless, the FWHM is more similar in the case of the three-component fit. This could be a direct consequence of the number of components. If we have two Gaussians for each emission line plus a broad component, its contribution to the global fit is going to be smaller than for a modeling of a single Gaussian plus a broad component, as the total flux of $\mathrm{H} \alpha-[\mathrm{N} \mathrm{II}]$ is going to be distributed among more components.

The number of components for the modeling of NGC 4594 and NGC 4736 is the same as C15 (see Table 3; Figs. B.6 and B.9), thus no more additional models were considered. In both cases, the results are in agreement with $\mathrm{C} 15$, although these authors indicate that the very broad component of NGC 4594 is very difficult to interpret (see Sect. 6.3.2). This very broad component dominates over the narrow to the $\mathrm{H} \alpha$ line flux in both analyses, as can be seen in Fig. B.6. The broad component for NGC 4736 contributes significantly to the total flux of both $\mathrm{H} \alpha$ and the whole $\mathrm{H} \alpha-[\mathrm{N}$ II] complex, as can be seen in Fig. 1.

For three galaxies (NGC 4374, NGC 4552 and NGC 4698; Figs. B.3, B.5, B.8, respectively) we derive a very broad component $\left(>1300 \mathrm{~km} \mathrm{~s}^{-1}\right)$ that $\mathrm{C} 15$ do not report in their analysis. According to our modeling, the fit is improved in the three galaxies by adding a very broad $\mathrm{H} \alpha$ component to the $\mathrm{H} \alpha-[\mathrm{N}$ II] complex. These detections are individually discussed in Sect. 6.3.2.

\section{Modeling of Palomar data}

After the summary of the overall modeling of the Palomar spectra, we compare the results with the work by Ho et al. (1997). All the results from the spectra modeling are found in Appendix A. 


\subsection{Overall modeling summary}

The Palomar data was analyzed by Ho et al. (1997) (hereinafter H97), who were also looking for the possible presence of a very broad component in the spectra. We analyzed eight galaxies from their sample by testing all three different fitting methods as described in Sect. 3. This is possible for the ground-based dataset, as the [O I] lines are visible in all cases (Table 3, Col. 4; see figures in Appendix B).

The best fitting is obtained with the S-method for nearly all the spectra (7 out of 8), as no significant improvement was seen when using the other methods. The galaxy NGC 4552 was the only one for which we selected the O-method, due to the severe blending of the [S II] lines (see Fig. B.5).

We find that four galaxies (NGC 2685, NGC 4552, NGC 4698, and NGC 4736) can be modeled with a single narrow component in all the emission lines, and three galaxies (NGC 3245, NGC 4374, and NGC 4486) need a narrow plus a secondary component, with NGC 4486 requiring a very broad component to obtain a proper model of the spectrum (see Sect. 6.3.1). Galaxy NGC 4594 is the only one whose spectrum is modeled with a narrow Gaussian component for all the forbidden lines and an additional broad $\mathrm{H} \alpha$ component (see Fig. B.6). In summary, a very broad component in $\mathrm{H} \alpha$ is needed in two out of the eight galaxies, and a secondary component is needed in three out of the eight LINERs (see Table 3).

\subsection{Comparison with Ho et al. (1997) (H97)}

In the search for broad emission lines carried out by H97, they analyzed the data of the galaxies from Ho et al. (1995), whose line profiles showed (or at least hinted at) the presence of a broad $\mathrm{H} \alpha$ emission. This reduced the total sample from 486 to 211 galaxies, from which only in 34 objects this broad feature was detected. The possible existence of a broad component in eight of the common objects is discussed below.

The methodology used in H97 to analyze the spectra consisted of an initial stellar subtraction using the spectra of galaxies devoid of emission lines (i.e., "template galaxies method", Ho et al. 1993, and references therein) and then a line modeling. The template galaxies method was also tested by $\mathrm{C} 18$, although when applying the PPXF routine to the spectra of the templates (see Sect. 3), some weak emission lines were visible in six out of the ten template galaxies. Thus, we used the PPXF method instead of the template galaxies method, as they need to be treated very carefully in order to avoid the inclusion of emission lines produced by the interstellar medium (see C18 for further details).

The line modeling in H97 consisted of a single Gaussian fit to the [S II] lines, considering them as reference to model the $\mathrm{H} \alpha-[\mathrm{N}$ II] lines. Similarly to our methodology, an additional broad component could be added to the $\mathrm{H} \alpha$ line only when necessary (see Sect. 3.2).

The spectra modeling for four of the galaxies (NGC 3245, NGC 4486, NGC 4594, and NGC 4698) is shown in H97. Three of them (namely NGC 4552, NGC 4594, and NGC 4698) are among their null detection of a broad component, whereas NGC 2685, NGC 3245, and NGC 4486 are among the ambiguous cases. For these, according to H97, the cause of ambiguity is the template chosen for the starlight subtraction.

In our analysis, thanks to the use of PPXF, we minimized possible source of ambiguity related to the stellar modeling and subtraction (see Sect. 3 and C18). In our modeling for these same spectra, we find a broad component in two out of the eight common objects (NGC 4486 and NGC 4594). Only for NGC 4594 is
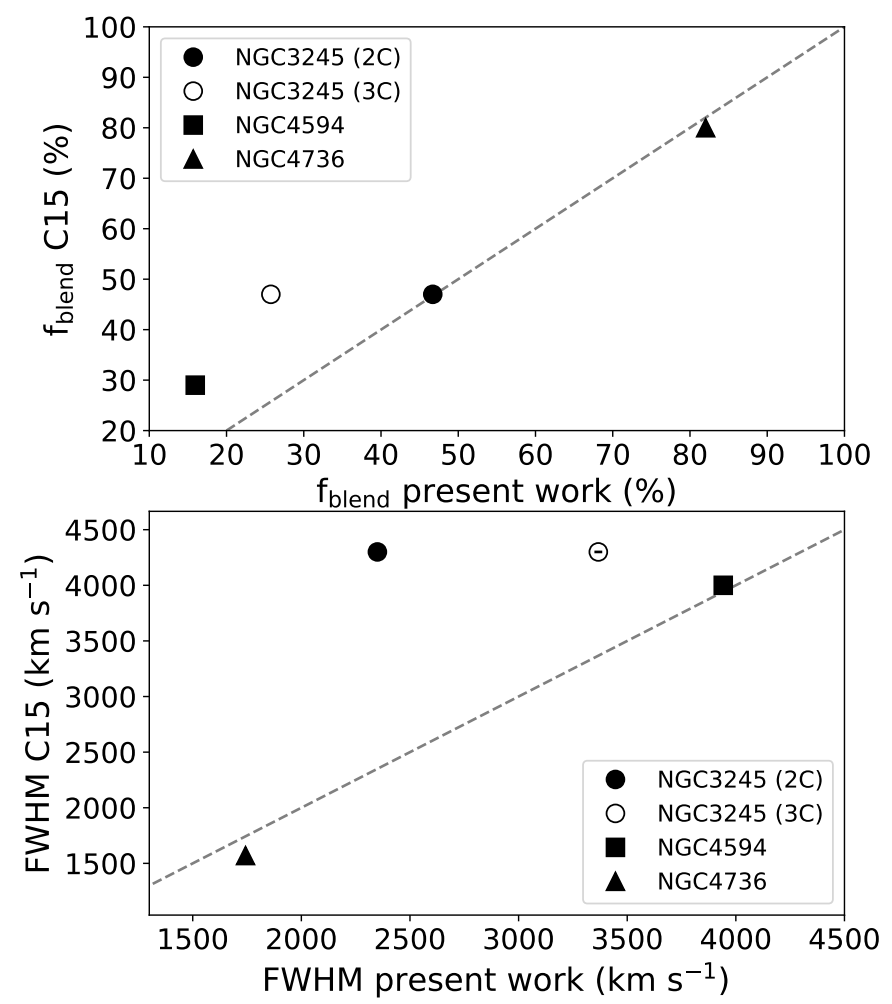

Fig. 1. Comparison of the contribution in percentage of the broad component of $\mathrm{H} \alpha$ to the total flux of the $\mathrm{H} \alpha-[\mathrm{N}$ II] complex (upper panel) and the FWHM of the very broad component (lower panel) derived in this work vs. C15. Each symbol represents a different LINER as indicated in the legend. The filled (empty) circle represents the two- (three-) component fit to NGC 3245. The gray dashed line is the one-to-one comparison.

this broad component also visible on the HST/STIS spectra (see Fig. B.6). This result indicates that the two objects, or at least NGC 4594, could initially have been classified as type 1 LINERs also with ground-based spectroscopy using a different starlight decontamination method and a different line modeling. For the other galaxy, NGC 4486, the existence of a broad component has been a matter of debate in previous works with both groundand space-based spectroscopy (e.g., Harms et al. 1994; Ho et al. 1997; Walsh et al. 2013; Constantin et al. 2015, see Sect. 6.3.1). It was described in $\mathrm{H} 97$ as showing weak wings at either side of [N II] lines. Although when adding a broad component to the fitting their residuals improve, they say that this component could be a product of some ambiguities with the modeling, so they decided not to include it in the analysis. Its contribution to the global fit is rather weak (5\%), but we also improve the residuals from our fitting by including it (Fig. B.4). We discuss its presence or absence in our ground and space data in Sect. 6.4.

\section{Results and discussion}

We note that, given the difference among the slit widths between Palomar and HST/STIS data (see Sect. 3.2), we consider the latter results as the most accurate in characterizing the innermost parts of the AGN. Hence, the following kinematical analysis is only focused on them. Apart from this, the possible discrepancies between the measured narrow-component velocities from ground- to space-based data (see Table 3) may be produced by the different alignment of the slits. 

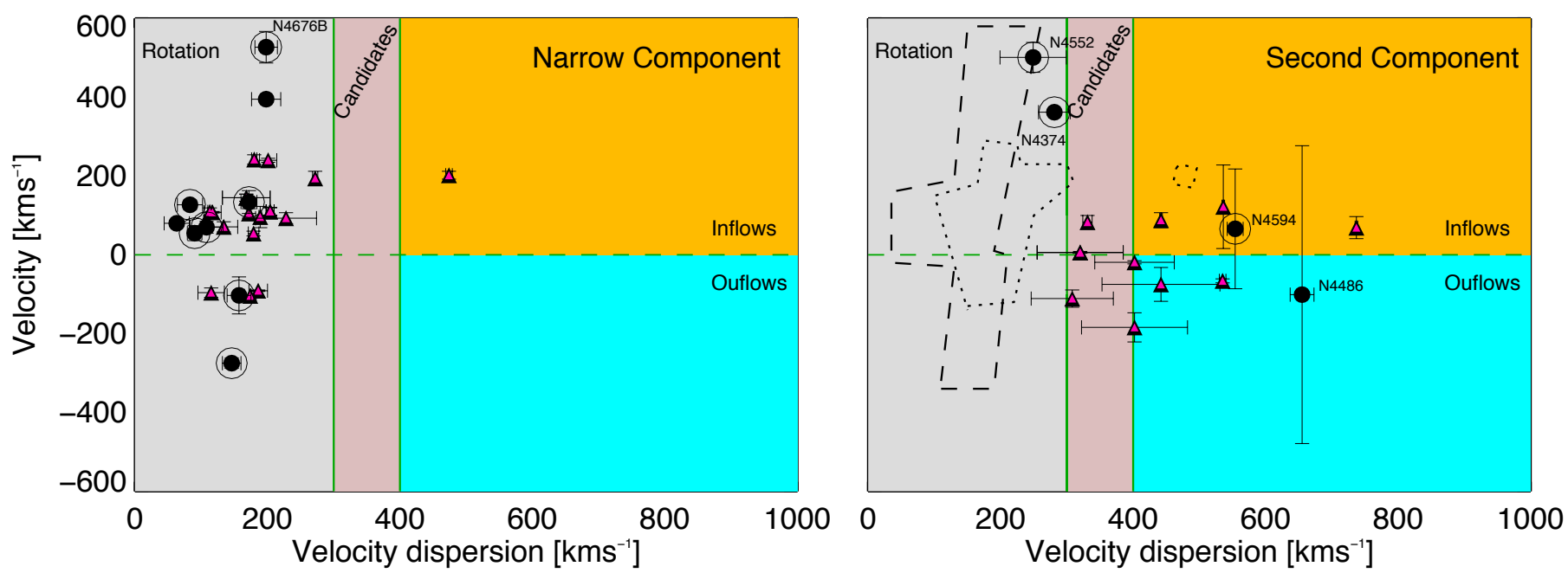

Fig. 2. Velocity dispersion vs. velocity derived for narrow (left panel) and secondary (right panel) components used to model the HST/STIS spectra for both type 1.9 from C18 (red triangles) and type 2 (black filled circles) LINERs. An additional black circle marks those cases for which a broad component is needed in HST/STIS spectra. All type 1.9 LINERs from C18 show a broad component. Each of the marked regions indicates to which kinematic component the modeled component can be associated. The orange region indicates inflows, the blue region indicates outflows, the pink region indicates candidates for both inflows and outflows, and the gray region indicates rotation. On the right panel, the contours of the narrow components are shown for both type 1.9 (dotted lines) and type 2 (dashed lines) LINERs.

\subsection{Overall summary of kinematics}

The wavelength-shift and line-width of each of the Gaussian components used to model the spectra are converted into a measurement of velocity and velocity dispersion of the ionized gas kinematics. We can associate them to a kinematic component of the galaxy. With this aim, we followed the velocity-velocity dispersion $(V-\sigma)$ classification similar to the one used in $\mathrm{C} 18$, which is shown in Fig. 2.

This figure is divided into four different regions, associated with rotation, outflows, inflows and candidates for rotational motions. Dividing limits of the regions have been established by $\mathrm{C} 18$ by measuring the gas velocity field from the $2 \mathrm{D}$ spectra of the type 1.9 LINERs within their sample, and estimating the maximum broadening of the emission lines associated with rotational motions. The $\mathrm{C} 18$ conservative upper limit for this component is $\sim 400 \mathrm{~km} \mathrm{~s}^{-1}$, although the typical value of velocity dispersion $\left(\sim 200 \mathrm{~km} \mathrm{~s}^{-1}\right)$ in their sample is well below this limit (see Sect. 5.2 in C18). This limit is supported by the fact that the ionized gas in rotation usually produces a broadening of the lines that is translated into velocity amplitudes up to $\sim 300 \mathrm{~km} \mathrm{~s}^{-1}$ (see e.g., Cappellari et al. 2007; Epinat et al. 2010). They found that the regions outside the nuclear zone in the $2 \mathrm{D}$ spectra had amplitudes $\sim 300-400 \mathrm{~km} \mathrm{~s}^{-1}$, thus associating this part of the $V-\sigma$ diagram to candidates for broadening produced by rotational motions.

As for the velocities, the narrow component of type 1.9 LINERs typically has values $\pm 50 \mathrm{~km} \mathrm{~s}^{-1}$. Thus, when the $\sigma$ is larger than $300 \mathrm{~km} \mathrm{~s}^{-1}$, these broadenings cannot be addressed as rotational motions, but instead as nonrotational motions. Then, we would consider them as candidates for the presence of outflows or inflows. For the intermediate cases, the targets are considered as candidates for nonrotational motions.

For the secondary component, $\mathrm{C} 18$ found that the velocity dispersion present typically values from 400 to $800 \mathrm{~km} \mathrm{~s}^{-1}$. In this case, the broadening is probably related to turbulent nonrotational motions. We can see in Fig. 2 that the velocities for the secondary component of type 1.9 LINERs present typical values ranging from $-200 \leq V_{\mathrm{S}} \leq 100 \mathrm{~km} \mathrm{~s}^{-1}$ (see C18). For this analysis, we decided to apply similar limits to $\mathrm{C} 18$, because the complete study of the gas velocity field could not be done with the present dataset. Thus, it has to be noted that the region limits could be different for type 2 LINERs if a similar analysis is considered. However, the physical interpretation of the position in the diagram for the galaxies with a secondary component is unlikely to change dramatically, given their large velocity dispersion $>500 \mathrm{~km} \mathrm{~s}^{-1}$, and hence their position on Fig. 2.

\subsection{Classification of the velocity components}

As mentioned in the previous section, if the velocity dispersion is in the range up to $300 \mathrm{~km} \mathrm{~s}^{-1}$, the component may be associated with the presence of a rotating disk in the galaxy. The broad $\mathrm{H} \alpha$ component is not considered in this figure, as it is interpreted unequivocally as a manifestation of the BLR of the AGN. The narrow component, present in all the ground- and space-based spectra, shows velocity dispersions consistent with being produced by rotation. On the other hand, the secondary component is always broader than the narrow one, and it has a higher spread on the velocity dispersion for both type 1.9 and type 2 LINERs, with typical values higher than $\sim 400 \mathrm{~km} \mathrm{~s}^{-1}$. These values are consistent with the presence of nonrotational motions (outflows or inflows).

In Fig. 2, only four galaxies of the HST/STIS sample (NGC 4374, NGC 4486, NGC 4552, and NGC 4594) needed a secondary component on the line modeling. From these, only for NGC 4374 and NGC 4486 is a second component added to the Palomar spectra. NGC 4486 and NGC 4594 line widths of the secondary component are consistent with being caused by the presence of nonrotational motions, which was also suggested by previous results (e.g., the $\mathrm{H} \alpha$ morphology of NGC 4486 shown in Masegosa et al. 2011). For the remaining cases, the line widths are compatible with being produced by rotational motions, although NGC 4374 could be a possible inflow candidate. Also in Masegosa et al. (2011), the possible presence of an outflow seen with the $\mathrm{H} \alpha$ emission was discarded (or inconclusive) for this galaxy due to the dust lanes that obscure and 
limit the access to the nuclear $\mathrm{H} \alpha$ morphology. For NGC 4552, a bipolar nuclear outflow was reported by Machacek et al. (2006) with Chandra images $(0.5-2 \mathrm{keV})$.

The other galaxy in the sample that has been previously reported as hosting an outflow is NGC 3245. The $\mathrm{H} \alpha$ image of this galaxy shows an unresolved nucleus and a structure indicative of the presence of an outflow (Masegosa et al. 2011), which is also supported by the kinematic analysis of (Walsh et al. 2008). However, in our modeling of the HST/STIS spectrum, a secondary component is not needed (see Sect. 6.3.2) and, if used, the resulting velocity and velocity dispersion would be compatible with being caused by rotation $\left(V_{\mathrm{s}}=-40 \pm 25 \mathrm{~km} \mathrm{~s}^{-1}\right.$; $\left.\sigma_{\mathrm{s}}=194 \pm 56 \mathrm{~km} \mathrm{~s}^{-1}\right)$. Using the Palomar data instead, a secondary component is clearly required to satisfactorily reproduce the line profiles with low residuals (Fig. B.2). In this case, the secondary component also has a velocity, and velocity dispersion in agreement with rotation, so it is not clear if it could be associated with nonrotational motions.

We can directly compare our values with the results from the HST/STIS analysis of 12 type 1.9 LINERs from C18. It is clear that the narrow component for both samples can be explained by rotational motions (see Fig. 2). The typical values of velocities and velocity dispersion (see Sect. 6.1) are similar except in some cases. Galaxy NGC $4676 \mathrm{~B}$ has a velocity higher than $500 \mathrm{~km} \mathrm{~s}^{-1}$. It is a merging galaxy, and its modeling is not wellconstrained (see Fig. B.7 and Sect. 6.3). In Wild et al. (2014), they quote a big velocity gradient oriented in a similar direction to where the HST/STIS slit is allocated, which may suggest that the velocity of the narrow component could be affected by this. Galaxies NGC 4374 and NGC 4486 have larger velocities of the narrow component than should be expected (see Sect. 6.1). For the latter, Noel-Storr et al. (2003) also found that the narrow component was not rest frame in the central spectrum. For the first, they might be produced by the large velocity gradients present in the innermost parts and that we are not considering individually in this analysis (see Sect. 6.3.2). On the other hand, the values for the secondary component are more spread throughout the diagram, including two galaxies in the range in which the broadening could be explained by rotation. Velocities for the secondary component of type 1.9 LINERs are more concentrated than those for type 2 LINERs, with values ranging from $-110 \leq V_{\mathrm{S}} \leq 500 \mathrm{~km} \mathrm{~s}^{-1}$ for the latter. The velocity dispersion range is more similar for both samples, ranging from $250 \leq V_{\mathrm{S}} \leq 800 \mathrm{~km} \mathrm{~s}^{-1}$. The proportion of objects within the $\mathrm{C} 18$ sample that present a secondary component is slightly higher than that of type 2 LINERs ( 7 out of 11 vs. 4 out of 9). This result could be a consequence of the orientation of the slit, which could not capture the full extension of putative outflows or inflows. However, this comparison could be not completely representative of these populations, due to the modest amount of type 2 LINERs in the sample.

\subsection{Individual comments for the galaxies}

This section is organized taking into account the components used to fit the HST/STIS spectra, as indicated in Table 3 (Col. 3). The figures with the spectra modeling are in Appendix B. As in all cases the BLR components in our analysis have large velocity shifts, we tried to set the velocity of the BLR component to zero in order to statistically confirm its true velocity. The majority of the models presented worse residuals both visually and statistically (i.e., difference in $\chi^{2}>15 \%$ worse) than with the final velocities (see Table 3 ). We discuss the results for the galaxies that did not change much with this model individually.

\subsubsection{Model with only narrow components}

As mentioned in Sect. 4, we have been able to model only two of the galaxies (NGC 2685 and NGC 4486) with a narrow or a narrow plus a secondary component (see Table 3 ).

The HST/STIS spectra of NGC 2685 has been analyzed in two works. González Delgado et al. (2004) compare the main ionizing mechanism of this LINER measured with both groundand space-based instruments, although the dataset for STIS use the G430L grating. It is the only galaxy in their analysis that requires a single narrow Gaussian component to reproduce the observed spectrum. C15 applied exactly the same modeling for the same dataset using only narrow components. Hence, our results are consistent with previous ones.

The galaxy NGC 4486 is a complex object with a wellknown radio jet. Noel-Storr et al. (2003) analyzed this spectrum initially and found that the presence of a broad component improved the global fit of the spectrum visually and statistically with respect to a single Gaussian component per line. The velocity of the narrow component they measure in the central pixel is $\sim 260 \mathrm{~km} \mathrm{~s}^{-1}$. Neither Walsh et al. (2013) nor C15 found a broad $\mathrm{H} \alpha$ component when modeling the emission lines. Walsh et al. (2013) reported the existence of velocity gradients in the innermost regions of the slit, as in the case of NGC 4374 (see Sect. 6.3.2). The velocity dispersion can be as high as $600 \mathrm{~km} \mathrm{~s}^{-1}$ in the central row, and it decays in the following regions. In our case, this structure is integrated in our spectrum. Our fit does not improve significantly by adding a very broad component to a two component fitting (only 5\%), thus, only two components were used. In this case, the secondary component is consistent with an outflow (see Table 3 and Fig. 2). However, a second component with a large width could be also the result of the strong gradient in the velocity dispersion reported by Walsh et al. (2013). For the Palomar spectrum, a broad component improves the fit by $18 \%$, although the $\varepsilon_{\mathrm{c}}$ was already smaller than $3 \varepsilon_{\text {line }}$ (see the residuals of the fit without the broad component in Fig. B.4). Given this and the unclear detection in previous works, we should consider the (non)detection in the Palomar (HST/STIS) spectrum as ambiguous. The nondetection of the HST/STIS data implies that this component, if it exists, should not be attributed to typical BLR clouds orbiting nearby the black hole, but instead to other phenomena on larger scales (e.g., inflows/outflows).

\subsubsection{Models with broad BLR-originated component}

The remaining seven galaxies were found to require a very broad component with the emission-line fitting analysis. The galaxy NGC 3245 was classified as an intermediate case between a LINER and a transition object (Ho et al. 1997). The HST/STIS data was firstly analyzed by Barth et al. (2001), who studied the dynamics of the ionized gas in this galaxy. They suggested the possible presence of a broad component in $\mathrm{H} \alpha$, which was modeled by C15 (see Sect. 4.2). Their emission line modeling consisted of three Gaussian profiles fitted to [N II]- $\mathrm{H} \alpha$ lines (tied in velocity but allowed to vary in $\sigma$ ). In our case, visual inspection indicates that only one narrow component is sufficient to fit the lines, although with a secondary component, the $\chi^{2}$ of the fit improves by approximately $16 \%$. We prioritized the simplest model that is able to describe the observed spectrum (S-method; see Fig. B.2). In either case, a broad component in $\mathrm{H} \alpha$ was necessary to model the line profiles.

The spectrum of NGC 4594 obtained with the TIGER $3 \mathrm{D}$ spectrograph shows some irregularities in its nucleus and a strong velocity gradient $\left(\sim 300 \mathrm{~km} \mathrm{~s}^{-1}\right)$ near the center (Emsellem $\&$ Ferruit 2000). However, as neither the HST or Palomar slits are 
located along the major kinematic axis of the galaxy (see Fig. B.6), we would not expect a strong line broadening caused by the ordinary disk rotation. The $\mathrm{H} \alpha$ emission is concentrated in the spiral arms and in the nuclear region of the galaxy (Masegosa et al. 2011). There has been some debate about the presence of a broad $\mathrm{H} \alpha$ component in the spectra of this galaxy. There are studies supporting both its presence (Kormendy et al. 1996; Walsh et al. 2008) and its absence (Ho et al. 1997; Nicholson et al. 1998). Therefore, a methodology that takes into account all the different possibilities, like the one presented in this work, is required to understand the true nature of this LINER nucleus. The spectrum presents wings in the $\mathrm{H} \alpha-[\mathrm{N} \mathrm{II}]$ lines that was initially attributed to the existence of a BLR with data from the HST/Faint Object spectrograph (Nicholson et al. 1998). On the one-to-one row analysis by Walsh et al. (2008), they show that there are large velocity gradients in the nuclear regions covered by the HST/STIS slit. These gradients could be producing the observed line profile, as indicated in Sect. 3.2. The best fit to the HST/STIS spectrum is obtained with two components plus a broad component, such as C15 (see Sect. 4.2 and Fig. B.6). The residuals are still higher than $3 \varepsilon_{\text {line }}$, they have been reduced from 3.2 to 2.7 for [N II] $\lambda 6548 \AA$, and the $\chi^{2}$ of the overall fit is improved by $15 \%$ by adding the broad component. If we were to set the velocity of the BLR component to zero, the $\chi^{2}$ would be only $3 \%$ worse, and the $\sigma$ would be $10 \mathrm{~km} \mathrm{~s}^{-1}$ less broad. However, as this component has a negligible flux contribution to the total $\mathrm{H} \alpha-[\mathrm{N} \mathrm{II]}$ blend, and it is the broadest line we found in our study (see Table 3), statistically we would expect to find no difference between both modelings. However, visually, the red wing of the [N II] $6584 \AA$ line is better reproduced with the velocity as indicated in Table 3 . Therefore, we decided not to set the velocity of the broad $\mathrm{H} \alpha$ component to zero.

The galaxy NGC 4736 is an interesting case, as the broad component is clearly visible in the HST/STIS spectrum (Constantin \& Seth 2012, C15), but it has never been reported from ground-based observations (Ho et al. 1997; Constantin \& Seth 2012). The size of the slit aperture is crucial in this object to classify it properly. The broad component has also been reported with a principal component analysis (PCA) tomography method applied to integral field unit (IFU) data taken several years after the HST/STIS spectra (Steiner et al. 2009). In our analysis, all the forbidden lines, except the $\mathrm{H} \alpha-[\mathrm{N}$ II] complex, are wellmodeled with a single narrow Gaussian component. For $\mathrm{H} \alpha$, a broad component of $\sigma_{\mathrm{B}} \sim 740 \mathrm{~km} \mathrm{~s}^{-1}$ is needed (see Sect. 4.2 and Fig. B.9). The FWHM of this component is within the limit of being associated with the BLR of the galaxy, which is also the case for NGC 4676B (see below). Both $\sigma_{\mathrm{B}}$ are only slightly higher $\left(\sim 100 \mathrm{~km} \mathrm{~s}^{-1}\right)$ that the $\sigma_{\mathrm{S}}$ for NGC 4486 .

$\mathrm{C} 15$ did not report additional very broad detections for the other galaxies in common (NGC 2685, NGC 4374, NGC 4486, and NGC 4552; see Sect. 4.2). However, in our analysis, two of them present a very broad component, whereas the other two only need a narrow, and a narrow plus a secondary component (NGC 2685 and NGC 4486, respectively), as previously indicated (see Sect. 6.3.1).

For the modeling of NGC 4374 and NGC 4552's nuclear spectra, we used a different technique from the rest of the galaxies. As explained in Sect. 3.2, in both cases the [O I] lines are barely detected, therefore only the S-method could be applied, but [S II] are severely blended.

The HST/STIS spectra of NGC 4374 were first analyzed by Bower et al. (1998), and a few years later by Walsh et al. (2010). In the former work, they used two Gaussian components to fit the lines, considering that they were tracing two dynamically different gas components, (a rotating disk and a low-velocity component unrelated to the disk). The latter showed that the profiles of [N II] $-\mathrm{H} \alpha$ could be affected by the rapidly rotating NLR kinematics, although their analysis does not exclude the presence of a broad component. As is the case of NGC 4594, the presence of a BLR-originated component is debated. In C15, they concluded that a broad component in $\mathrm{H} \alpha$ was not needed (see Sect. 4.2). Nevertheless, its presence was not ruled out by Walsh et al. (2010) in the three innermost rows, where a peaked central velocity dispersion, associated with the emission-line disk, is related to large velocity gradients visible mainly in the central row of the slit (see Sect. 6.2). In our analysis, these gradients are integrated in the final spectrum, as we did not perform a oneto-one row analysis (to maximize the $\mathrm{S} / \mathrm{N}$ ). In this sense, our narrow component fit should be properly considered as a lower limit to the velocity dispersion of the disk. Both narrow and secondary components are compatible with rotation (see Fig. 2 and Table 3), which is somewhat expected from the results of Walsh et al. (2010). In this work, they do not show the values of the three nuclear rows' analyses, as their kinematical profiles are said to be difficult to analyze due to the large gradients. However, the amplitude of the velocities is comparable with that of the secondary component $\left(\sim 400 \mathrm{~km} \mathrm{~s}^{-1}\right)$, being coincident with an assumed limit for detecting outflows (Sect. 6.2 and Fig. 2).

In the case of NGC 4552, Cappellari et al. (1999) modeled the emission lines with intermediate width profiles in both permitted and forbidden lines and highlighted the low luminosity of the central engine in comparison with other AGNs. With respect to the HST/STIS spectra, C15 did not consider a broad component. Our modeling, which takes into account a second component in forbidden lines and narrow $\mathrm{H} \alpha$, reveals the presence of a broad component in both galaxies that contributes significantly ( $\sim 65 \%$ and $\sim 70 \%$, respectively) to the global fit, as can be seen in Figs. B.3 and B.5 (see Sect. 4.2).

The NGC 4698 data come from the Survey of Nearby Nuclei with STIS, known as SUNNS, analyzed by Shields et al. (2007). They suggest that the galaxy was erroneously classified as a type 2 Seyfert galaxy by Ho et al. (1997), on the basis of a decrease in the level of the excitation mechanism, suggesting that it is a LINER or a transition object. These authors did not detect a broad component in $\mathrm{H} \alpha$ in their spectral analysis, as Balmaverde \& Capetti (2013) and C15 did when analyzing the same dataset in contrast to our findings. More specifically, we find that, even though the [S II] lines are perfectly modeled with a single narrow component, the addition of a weak broad component improves the $\chi^{2}$ of the fit $\sim 17 \%$ (see Fig. B.8). The lack of detection of this component in previous works could be due to its low contribution to the global fit (39\%, Table 3) flux in comparison with the narrow component (see Sect. 4.2). If we were to set the velocity of the broad component to zero, we find that the $\chi^{2}$ of the fit would be only $0.5 \%$ worse than the fit with the velocity from Table 3 . As for NGC 4594, the broad component has a rather low contribution to the global flux and is the second broadest component in our analysis, thus statistically we should again expect a small difference. In this case, visually there was also no difference. However, due to the rather low velocity of this component in the previous model ( $273 \pm 97 \mathrm{~km} \mathrm{~s}^{-1}$; Table 3$)$, we decided not to add any constraint to the broad component velocity.

The remaining galaxy, NGC 4676B, belongs to the Mice galaxies complex, two interacting spiral galaxies. Both spirals have been told to present features indicative of the presence of outflows (Masegosa et al. 2011; Wild et al. 2014). Its HST/STIS nuclear spectrum has not been studied before, as the $\mathrm{S} / \mathrm{N}$ is rather 
low, which makes it difficult to analyze (see Fig. B.7). Despite this, we attempted to model the emission line, that being the unique measurement of this kind for this object with HST/STIS data. Our fit reveals the presence of a broad component for the $\mathrm{H} \alpha-[\mathrm{N} \mathrm{II}]$ complex, while a narrow component is sufficient to fit the [S II] lines. Even though the $\chi^{2}$ improved with the addition of this component, the residuals are inside the $3 \varepsilon_{\text {line }}$ limits also with a single narrow modeling for all the lines. The presence of this component could be affected by the noise, and the FWHM of this component is not as broad $\left(748 \mathrm{~km} \mathrm{~s}^{-1}\right.$; Table 3$)$ as the other detections in our sample. The results of the test of setting the velocity of the broad component to a zero velocity show the same results in FWHM and $\varepsilon$-indicator. So, it is not clear if it can be associated with the presence of a BLR. To avoid possible ambiguities, in what follows, we have conservatively excluded it as a true detection of a broad component.

\subsection{BLR in optically classified type 2 LINERs}

Type 2 LINERs have been classified by optical, ground-based spectroscopy as not having a broad component in the Balmer emission lines, while this is not the case for type 1-1.9 (Ho et al. 1993, 1995). Nevertheless, we see in our analysis (Sect. 6.3) that six out of the nine analyzed galaxies in our sample exhibit this component in space-based spectroscopy. In the case of the 22 type 1.9 LINERs (selected from the Palomar Sky Survey), all the available space-based spectra (11 LINERS) showed a broad component in $\mathrm{H} \alpha$ (see $\mathrm{C} 18$ for details).

However, the same objects analyzed with the original Palomar spectra from Ho et al. (1997) do not show this feature, except for two cases in type 2 LINERs (NGC 4486 and NGC 4594), and they do show it in all but four cases in type 1.9 LINERs. Ho's initial classification is mainly supported by this result. However, this enhances the difference of the groundbased spectra with those from space, also based on the results of C18.

The detection of a broad component is favored in spacebased spectra because of the spatial resolution (Molina et al. 2018). If the BLR exists in a particular galaxy, a wider slit on a ground-based instrument could prevent it from being detected, as the weak broad $\mathrm{H} \alpha$ component could be diluted. This could be the reason for the initial lack of detection of the BLR in the galaxies of our sample, but also the modeling of the lines (e.g., testing single and multiple components) could be crucial to highlighting its presence (as was our case for HST/STIS spectra of NGC 4374 and NGC 4552).

The galaxy NGC 4486 is the only case in which the broad component is detected on the Palomar spectrum but not in the HST data. As we should expect exactly the opposite behavior (i.e., the detection of this component on HST and not in Palomar spectrum), it is not clear if this galaxy should be reclassified (type 1 instead of type 2). Moreover, if we take into account that the existence of the BLR in this object has been largely discussed in the literature (see Sect. 5.2). On the other hand, NGC 4594 is the only object in which a very broad component is detected in both datasets, so it should then be considered as a type 1 LINER.

\subsection{Line ratios}

We analyzed the line ratios for the emission lines available in all spectra. In Fig. 3, the [N II]/H $\alpha$ and [S II]/H $\alpha$ line ratios for both
Palomar and HST data are shown ${ }^{3}$. We estimated the ratios for both the narrow and the secondary components (when present) for all galaxies except NGC 4676B, as its modeling is not wellconstrained (see Sect. 6.3).

We consider, in Fig. 3, the empirically derived separation between an AGN or a star forming (SF) system, like in the BPT diagrams (Baldwin et al. 1981; Kauffmann et al. 2003; Kewley et al. 2006), based only in [N II] $/ \mathrm{H} \alpha$ and $[\mathrm{S} \mathrm{II}] / \mathrm{H} \alpha$ ratios. Since $[\mathrm{O} \mathrm{III]} / \mathrm{H} \beta$ is not available in our data, we consider the star formation as the dominant mechanism of ionization if $\log ([\mathrm{S} \mathrm{II}] / \mathrm{H} \alpha)$ is $<0.0$. The mechanism would be the AGN ionization when $\log ([\mathrm{S} \mathrm{II}] / \mathrm{H} \alpha)>0.0$. For the $\log ([\mathrm{N} \mathrm{II}] / \mathrm{H} \alpha)$, the limit for SF ionization is $<-0.2$, for $\mathrm{AGN}$ it is $>0.2$, and we consider it as a "transition" region elsewhere.

In general, evident differences are seen in both panels of Fig. 3 between the space- and ground-based data. For both $[\mathrm{N} \mathrm{II}] / \mathrm{H} \alpha$ and $[\mathrm{S} \mathrm{II}] / \mathrm{H} \alpha$, the ratios are always positive for the HST data, whereas more dispersion in the values exists in Palomar data. Specifically, for Palomar (HST) the average and standard deviation are, for $\log ([\mathrm{N} \mathrm{II}] / \mathrm{H} \alpha)$, equal to 0.15 and 0.30 (0.54 and 0.40), respectively; for $\log ([\mathrm{S} \mathrm{II}] / \mathrm{H} \alpha)$, these values are 0.02 and 0.24 (0.37 and 0.26$)$.

In the absence of $[\mathrm{O} \mathrm{III}] / \mathrm{H} \beta$ (see Sect. 2), the ratios are more compatible with the AGN region for the HST spectra than for the Palomar data. The difference could be due to the spatial resolution based on the recent results from Molina et al. (2018). They have analyzed three LINERs with HST data, and performed a detailed study on the line diagnostic diagrams considering the unresolved nuclear source and what they call an "integrated" spectrum, which resembles the spectra of the targets as seen from a ground-based telescope. They found that the line ratios tend to be higher for the unresolved nuclear source than for the integrated spectrum. They conclude that it could be produced by the addition of a large spatial region of the galaxy $(\sim 100 \mathrm{pc})$ within the integrated spectrum, where there is influence from gas probably suffering simultaneously photoionization and shock effects. This is reflected on their line ratios being consistent with different ionization mechanisms.

In Fig. 3, NGC 2685, NGC 4698, and NGC 4736 only have a single narrow component (black circles), so they appear only once. The difference compared to the one-to-one line could be explained directly with the variation of the $\mathrm{H} \alpha$ profile seen in the HST with respect to the Palomar spectra (see Figs. B.1, B.8 and B.9). Although, except for NGC 4698, they are compatible to being produced by an AGN in both Palomar and HST. For the case of NGC 4698, line ratios correspond to the transition region in both diagrams for the HST data, so this results in an ambiguous classification. The varying $\mathrm{H} \alpha$ profile does not only produce considerable differences in the line ratios for these three objects, but also for NGC 3245 and NGC 4486 (see Figs. B.2 and B.4, respectively).

The differences in the $\mathrm{H} \alpha$ profiles are significant, but there are also dramatic variations for the ratios of the secondary with respect to the narrow component. The most extreme case is NGC 4594 (see Figs. 3 and B.6), in which the $\mathrm{H} \alpha$ contribution to the total flux of the narrow component is almost negligible in comparison to the contribution of the secondary and the broad

$3[\mathrm{OI}] / \mathrm{H} \alpha$ is not considered, as the [O I] line is not available for all HST spectra (Table 3, Col. 4). 
component. However, the secondary component shows a very similar line ratio to the Palomar spectrum.

In the top panel of Fig. 3, considering the $[\mathrm{S} \mathrm{II}] / \mathrm{H} \alpha$ ratio, the great majority of points for ground-based data are located around the transition region (i.e., $\log ([\mathrm{S} \mathrm{II}] / \mathrm{H} \alpha) \sim 0.0)$. In these cases, the information of the $[\mathrm{O} I I I] / \mathrm{H} \beta$ ratio is required to further constraint the source of the ionization field. This is true for all objects except for NGC 4736, which is the only galaxy for which both $[\mathrm{N} \mathrm{II}] / \mathrm{H} \alpha$ and $[\mathrm{S} \mathrm{II}] / \mathrm{H} \alpha$ line ratios of the narrow component are consistent with being produced by the AGN, as mentioned above. Our classification, based on the $[\mathrm{S} \mathrm{II}] / \mathrm{H} \alpha$ ratio, is consistent with the presence of a very broad component (BLRoriginated) visible in its HST spectrum (Fig. B.9). On the other hand, for the classification based on $[\mathrm{N} \mathrm{II}] / \mathrm{H} \alpha$ ratio, the majority of objects ( 7 out of 8 ) fall within the AGN region, and only one is consistent with being ionized by a SF region.

\section{Summary and conclusions}

In this work, we present a kinematic analysis and the study of ionization mechanisms of the gas of nine type 2 LINERs with both space- and ground-based spectroscopy obtained from the HST/STIS archive and from the Palomar/Double Spectrograph (Ho et al. 1995), respectively. The main objective is the detection of the existence of a BLR in these galaxies by analyzing its spectral emission line features, and the possible presence of outflows. Moreover, we addressed the line ratios of the emission lines to study the dominant ionization mechanism for these objects.

We used the [S II] and [O I] lines as templates to model the other forbidden lines ([N II]) and narrow $\mathrm{H} \alpha$, and considered the addition of a broad component under $\mathrm{H} \alpha$ where necessary. We used a maximum of three Gaussian components called the narrow, secondary, and broad components. Our approach allowed us to explore a larger number of scenarios for modeling emission lines with respect to previous works. These scenarios include possible NLR stratification and nonrotational motions being present, and never explored, for type 2 LINER nuclei (see C18 for type 1 LINERs).

The narrow component is likely to be associated with the rotation from the galaxy disk, whereas the secondary component, of intermediate width, could be related to both rotational and nonrotational motions (as outflows). The broad component of Balmer lines is always associated with the BLR of the AGN.

The secondary component has been detected for four LINERs in the HST spectra and for three in the Palomar data. For two objects from the HST analysis (i.e., NGC 4486 and NGC 4594), this component is found to suggest nonrotational motions, such as outflows. The line width of these components is similar to those found for outflows in type 1.9 LINERs by Cazzoli et al. (2018).

The detection of the BLR in LINERs is crucial to revealing its true AGN nature. Ho et al. (1997) optically classified eight of the galaxies as type 2 objects, because their line fitting did not require a very broad BLR-originated component to reproduce the $\mathrm{H} \alpha-[\mathrm{N} \mathrm{II}]$ profile. With our analysis (following C18) of the same Palomar-spectra, we find that for two out of eight LINERs, a broad component is indeed necessary to reproduce emission lines. For the space-based data, a very broad component is needed in six out of the nine analyzed objects.

The only detection of this component in the Palomar data but not confirmed with the HST is for NGC 4486. The results for this component are not well-constrained, thus we consider the BLR component detection as ambiguous. The galaxy NGC 4594 is the
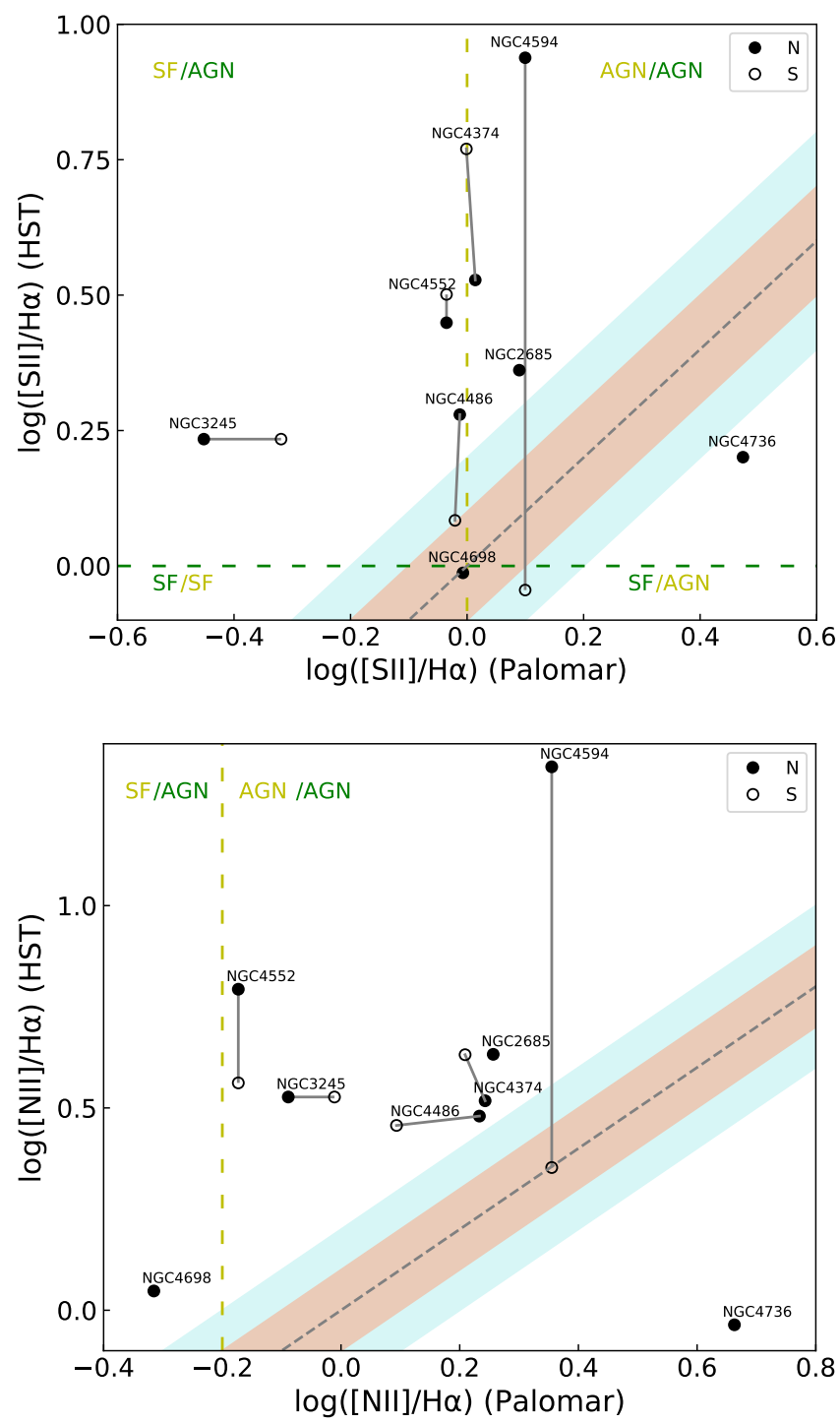

Fig. 3. Comparison of line ratios for $[\mathrm{S} \mathrm{II}] / \mathrm{H} \alpha$ (top) and $[\mathrm{N} \mathrm{II}] / \mathrm{H} \alpha$ (bottom) for both HST/STIS spectra ( $y$-axis) and Palomar spectra ( $x$-axis). The gray dashed line is the one-to-one comparison. Filled circles represent the narrow component, whereas the empty circles are for the secondary, which is connected to the narrow through a gray solid line when present. The light pink and light blue ranges indicate the $10 \%$ and $20 \%$ uncertainty levels with respect to the one-to-one comparison, respectively. Green and yellow dashed lines indicate the limits (HST and Palomar, respectively) for the star forming (SF) and AGN regions. The limits were obtained from Kauffmann et al. (2003) and Kewley et al. (2006).

only one in the sample for which a broad component is needed in both space- and ground-based spectra, indicating that it would be better classified as as a type 1 LINER.

Three of the BLR detections in the HST data (NGC 3245, NGC 4594, and NGC 4736) were already reported by Constantin et al. (2015). Our measurements of both FWHM and flux contribution of the broad component with respect to the total flux of the $\mathrm{H} \alpha-[\mathrm{N} \mathrm{II}]$ complex agree with Constantin et al. (2015).

The detection of a very broad component is favored with the HST data as the spatial resolution being ten times better than for the Palomar data, decreasing the amount of contaminant starlight from the host galaxy, and preventing the possible dilution of the broad component. The spatial resolution could be also relevant 
to the line diagnostic diagrams, as the calculated line ratios vary significantly from ground- to space-based spectra.

Acknowledgements. Authors acknowledge the anonymous referee for his/her constructive comments to improve the paper. We acknowledge financial support from the Spanish Ministerio de Ciencia, Innovación y Universidades (MCIU) under the grant AYA2016-76682-C3, and from the State Agency for Research of the Spanish MCIU through the "Center of Excellence Severo Ochoa" award to the Instituto de Astrofísica de Andalucía (SEV-2017-0709). LHM acknowledge financial support under the grant BES-2017-082471. We acknowledge O. Reyes-Amador for his useful help on this work. This research has made use of the NASA/IPAC Extragalactic Database (NED), which is operated by the Jet Propulsion Laboratory, California Institute of Technology, under contract with the National Aeronautics and Space Administration. We acknowledge the usage of the HyperLeda database (http://leda.univ-lyon1.fr). This work has made extensive use of IRAF and Python, particularly with ASTROPY (http:// WWW . astropy . org) (Astropy Collaboration 2013, 2018), MATPLOTLIB (Hunter 2007), NUMPY and LMFIT.

\section{References}

Astropy Collaboration (Price-Whelan, A. M., et al.) 2018, AJ, 156, 123 Astropy Collaboration (Robitaille, T. P., et al.) 2013, A\&A, 558, A33 Baldwin, J. A., Phillips, M. M., \& Terlevich, R. 1981, PASP, 93, 5

Balmaverde, B., \& Capetti, A. 2013, A\&A, 549, A144

Balmaverde, B., \& Capetti, A. 2014, A\&A, 563, A119

Barth, A. J., Sarzi, M., Rix, H.-W., et al. 2001, ApJ, 555, 685

Binette, L., Magris, C. G., Stasińska, G., \& Bruzual, A. G. 1994, A\&A, 292 13

Bower, C. A., Wilson, A. S., Heckman, T. M., \& Richstone, D. O. 1996, in The Physics of Liners in View of Recent Observations, eds. M. Eracleous, A. Koratkar, C. Leitherer, \& L. Ho, ASP Conf. Ser., 103, 163

Bower, G. A., Green, R. F., Danks, A., et al. 1998, ApJ, 492, L111 Cappellari, M. 2017, MNRAS, 466, 798

Cappellari, M., \& Emsellem, E. 2004, PASP, 116, 138

Cappellari, M., Renzini, A., Greggio, L., et al. 1999, ApJ, 519, 117

Cappellari, M., Emsellem, E., Bacon, R., et al. 2007, MNRAS, 379, 418

Cazzoli, S., Márquez, I., Masegosa, J., et al. 2018, MNRAS, 480, 1106

Constantin, A., \& Seth, A. C. 2012, Adv. Astron., 2012, 178060

Constantin, A., Shields, J. C., Ho, L. C., et al. 2015, ApJ, 814, 149

Dopita, M. A., \& Sutherland, R. S. 1995, ApJ, 455, 468

Dopita, M. A., Koratkar, A. P., Evans, I. N., et al. 1996, in The Physics of Liners in View of Recent Observations, eds. M. Eracleous, A. Koratkar, C. Leitherer \& L. Ho, ASP Conf. Ser., 103, 44

Emsellem, E., \& Ferruit, P. 2000, A\&A, 357, 111

Epinat, B., Amram, P., Balkowski, C., \& Marcelin, M. 2010, MNRAS, 401, 2113
González Delgado, R. M., Cid Fernandes, R., Pérez, E., et al. 2004, ApJ, 605, 127

González-Martín, O., Masegosa, J., Márquez, I., Guainazzi, M., \& JiménezBailón, E. 2009, A\&A, 506, 1107

Groves, B. A., Dopita, M. A., \& Sutherland, R. S. 2004, ApJS, 153, 75

Harms, R. J., Ford, H. C., Tsvetanov, Z. I., et al. 1994, ApJ, 435, L35

Heckman, T. M. 1980, A\&A, 87, 152

Ho, L. C. 2008, ARA\&A, 46, 475

Ho, L. C., Filippenko, A. V., \& Sargent, W. L. W. 1993, ApJ, 417, 63

Ho, L. C., Filippenko, A. V., \& Sargent, W. L. 1995, ApJS, 98, 477

Ho, L. C., Filippenko, A. V., \& Sargent, W. L. W. 1997, ApJS, 112, 315

Ho, L. C., Filippenko, A. V., \& Sargent, W. L. W. 2003, ApJ, 583, 159

Ho, L. C., Greene, J. E., Filippenko, A. V., \& Sargent, W. L. W. 2009, ApJS, 183, 1

Huchtmeier, W. K. 1994, A\&A, 286, 389

Huchtmeier, W. K., \& Richter, O.-G. 1986, A\&AS, 64, 111

Hunter, J. D. 2007, Comput. Sci. Eng., 9, 90

Kauffmann, G., Heckman, T. M., Tremonti, C., et al. 2003, MNRAS, 346, 1055

Kewley, L. J., Groves, B., Kauffmann, G., \& Heckman, T. 2006, MNRAS, 372 961

Kormendy, J., Bender, R., Ajhar, E. A., et al. 1996, ApJ, 473, L91

Machacek, M., Nulsen, P. E. J., Jones, C., \& Forman, W. R. 2006, ApJ, 648, 947 Márquez, I., \& Moles, M. 1996, A\&AS, 120, 1

Márquez, I., Durret, F., González Delgado, R. M., et al. 1999, A\&AS, 140,

Márquez, I., Masegosa, J., Durret, F., et al. 2003, A\&A, 409, 459

Márquez, I., Masegosa, J., González-Martin, O., et al. 2017, Front. Astron. Space Sci., 4, 34

Masegosa, J., Márquez, I., Ramirez, A., \& González-Martín, O. 2011, A\&A, 527, A23

McKernan, B., Ford, K. E. S., \& Reynolds, C. S. 2010, MNRAS, 407, 2399

Molina, M., Eracleous, M., Barth, A. J., et al. 2018, ApJ, 864, 90

Nicholson, K. L., Reichert, G. A., Mason, K. O., et al. 1998, MNRAS, 300, 893

Noel-Storr, J., Baum, S. A., Verdoes Kleijn, G., et al. 2003, ApJS, 148, 419

Oke, J. B., \& Gunn, J. E. 1982, PASP, 94, 586

Osterbrock, D. E., \& Ferland, G. J. 2006, Astrophysics of Gaseous Nebulae and Active Galactic Nuclei, 2nd edn. (Sausalito: University Science Books)

Papaderos, P., Gomes, J. M., Vílchez, J. M., et al. 2013, A\&A, 555, L1

Pogge, R. W., Maoz, D., Ho, L. C., \& Eracleous, M. 2000, ApJ, 532, 323

Shields, J. C., Rix, H.-W., Sarzi, M., et al. 2007, ApJ, 654, 125

Stasińska, G., Vale Asari, N., Cid Fernandes, R., et al. 2008, MNRAS, 391, L29

Steiner, J. E., Menezes, R. B., Ricci, T. V., \& Oliveira, A. S. 2009, MNRAS, 395, 64

van Dokkum, P. G. 2001, PASP, 113, 1420

Walsh, J. L., Barth, A. J., Ho, L. C., et al. 2008, AJ, 136, 1677

Walsh, J. L., Barth, A. J., \& Sarzi, M. 2010, ApJ, 721, 762

Walsh, J. L., Barth, A. J., Ho, L. C., \& Sarzi, M. 2013, ApJ, 770, 86

Wardle, M., \& Knapp, G. R. 1986, AJ, 91, 23

Wild, V., Rosales-Ortega, F., Falcón-Barroso, J., et al. 2014, A\&A, 567, A132 


\section{Appendix A: Stellar modeling and subtraction for \\ Palomar spectra}

The starlight modeling and subtraction to the ground-based spectra is presented here for the eight available LINERs. The stellar

continuum in the observed spectra was modeled using the PPXF method as it was in Cazzoli et al. (2018) (Sect. 3.1).

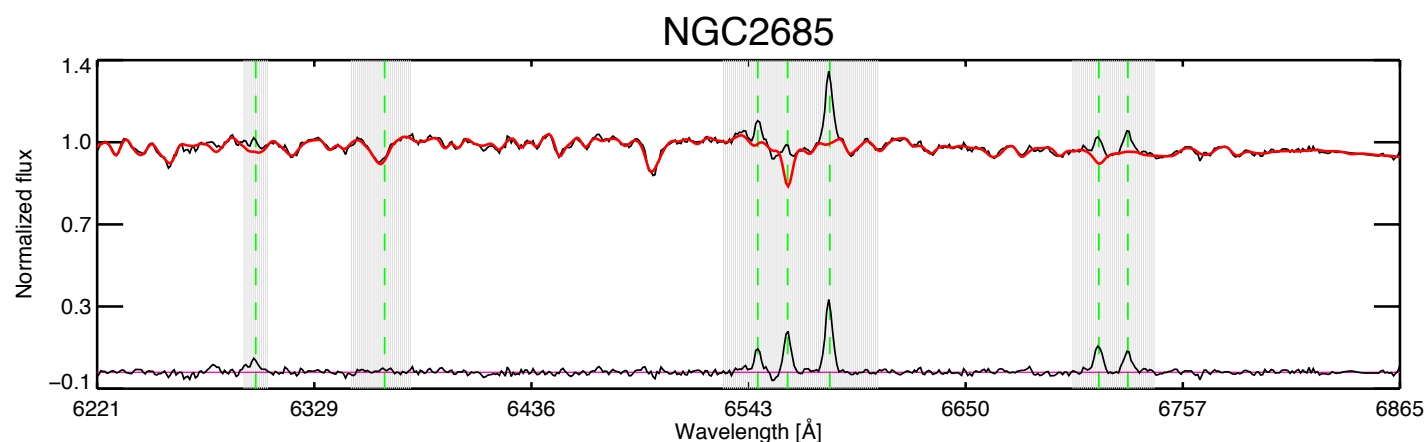

Fig. A.1. Optical ground-based Palomar spectrum of NGC 2685. The original data is shown in black; the red line indicates the modeled stellar spectrum that matches the observed stellar continuum. The spectrum used for the kinematical decomposition (i.e., data - model) is also presented. Green dashed lines show the rest-frame position of the [O I], [N II]-H $\alpha$, and [S II] lines. Gray bands indicate the regions masked to perform the stellar modeling. The magenta line indicates the zero level as reference.

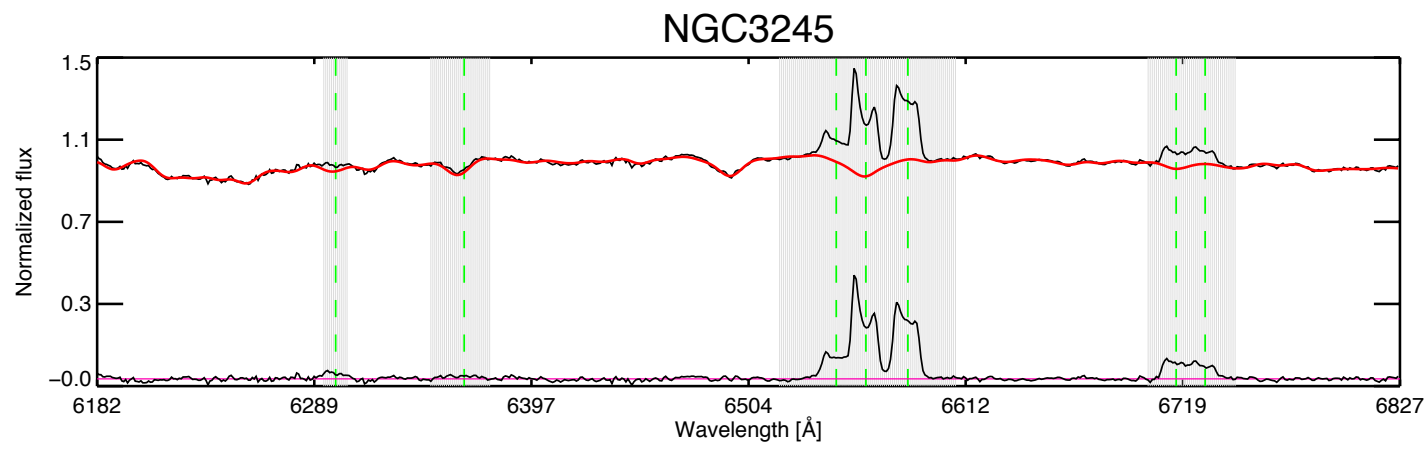

Fig. A.2. Same as Fig. A.1 but for NGC 3245.

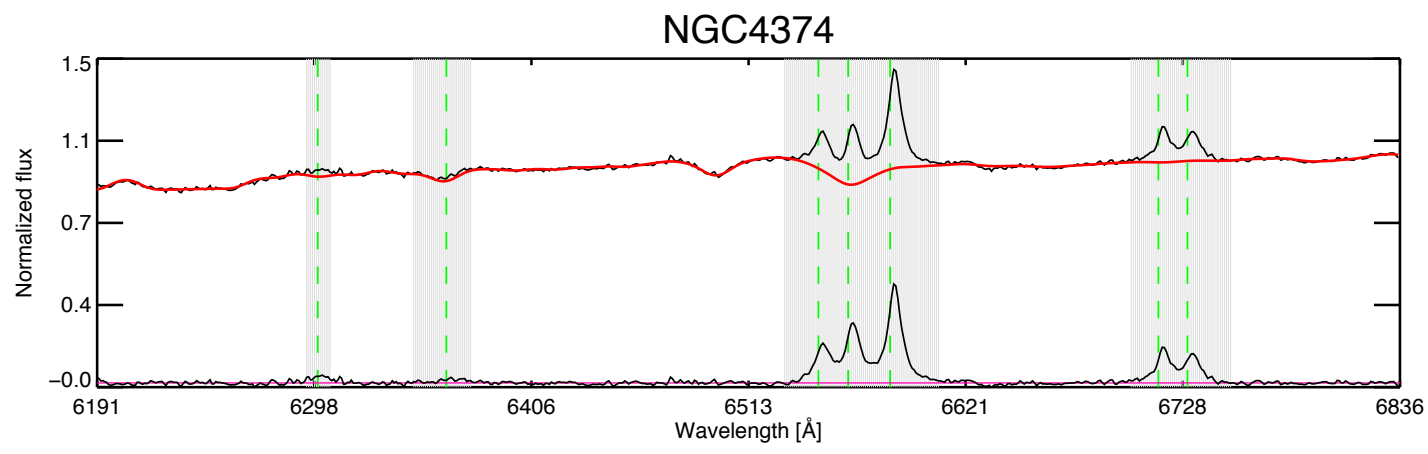

Fig. A.3. Same as Fig. A.1 but for NGC 4374.

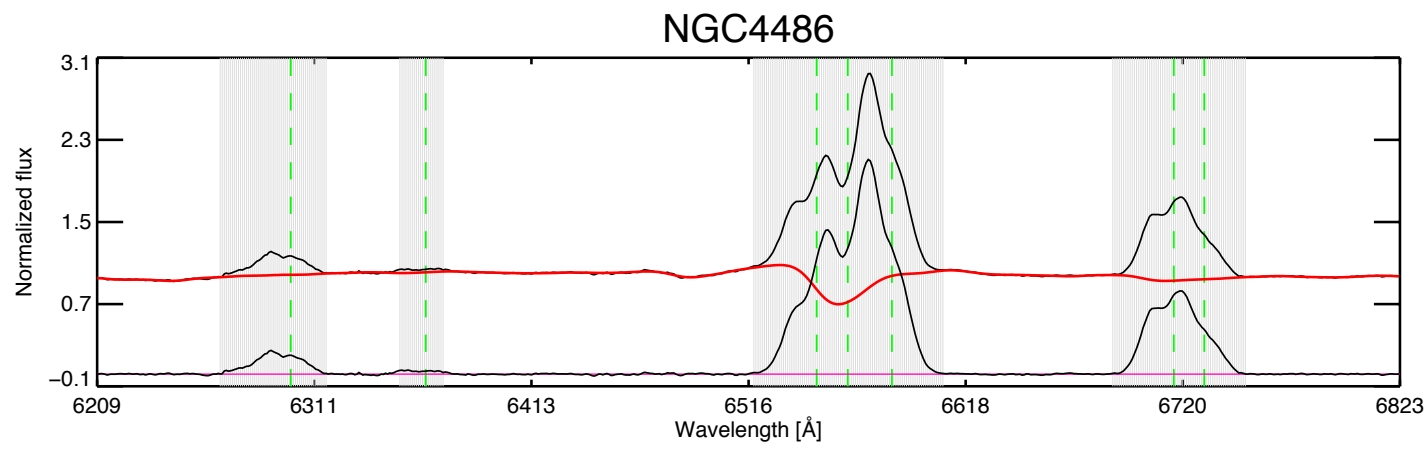

Fig. A.4. Same as Fig. A.1 but for NGC 4486. 


\section{NGC4552}

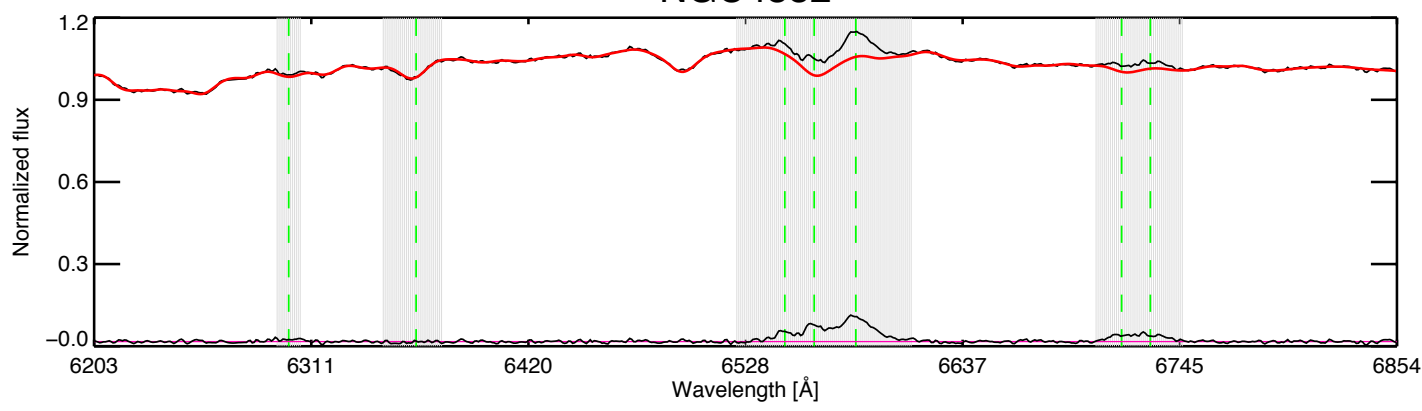

Fig. A.5. Same as Fig. A.1 but for NGC 4552.

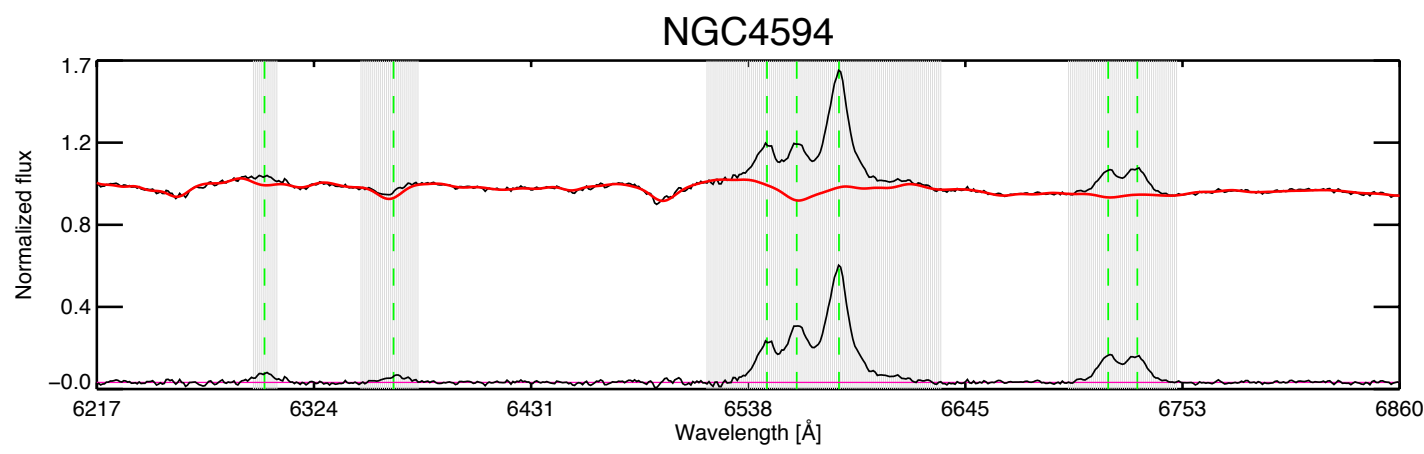

Fig. A.6. Same as Fig. A.1 but for NGC 4594.

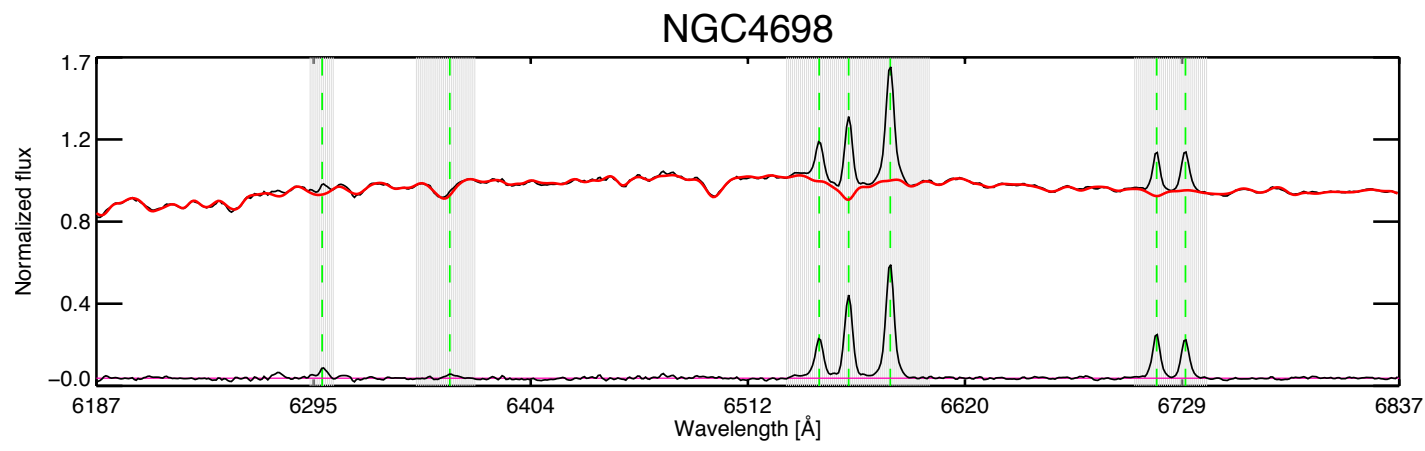

Fig. A.7. Same as Fig. A.1 but for NGC 4698.

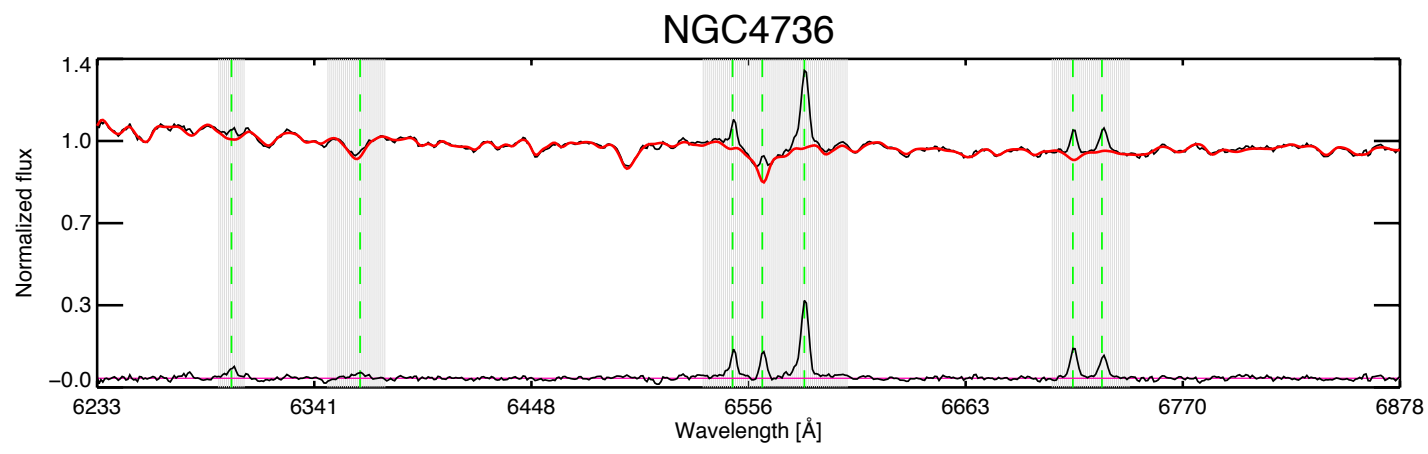

Fig. A.8. Same as Fig. A.1 but for NGC 4736. 


\section{Appendix B: Spectra modeling for each galaxy}

We present HST images from the archive and the spectra analyzed in this manuscript for each LINER. They are arranged as follows:

Top. Sharp divided (Márquez \& Moles 1996; Márquez et al. $1999,2003)$ HST image of the galaxy in the inner $10^{\prime \prime} \times 10^{\prime \prime}$. The HST filters are summarized in Table 2, Col. 9. Sharp-divided images are very useful for tracing asymmetries in the light distribution, such as bars, spiral arms, dust lanes, and rings. The method consists of dividing the original image by that obtained from the convolution with a median filter of 30 pixels. This technique allows the subtraction of the diffuse background emission facilitating the detection of small-scale variations and rouses the notion of a possible presence of both dust extinguished, and more luminous regions (e.g., Masegosa et al. 2011). The galaxy ID is indicated in the panel title. White and yellow continuous lines represent the slit used to obtain the HST/STIS and Palomar spectrum (when available), respectively. The angular scale of $1^{\prime \prime}$ of the image in parsecs (see Table 1, Col. 6) is indicated in the right-upper part of the image. The white dashed line indicates the PA of the major axis (see Table 1).

Middle. Line modeling of the HST/STIS spectra (see Sect. 3 for details). We marked each Gaussian component with different colors (identical colours mark the same kinematic components). Green lines represent the narrow component; when present, blue lines represent the secondary component, and purple lines, the broad component. The red curve shows the total contribution of all the components. The continuum range selected to calculate the standard deviation is plotted in yellow. Residuals from the fits are in the middle/lower panels. We have included the residuals of the fit without the broad component for those objects in which this component was necessary (labeled as: "Res 1") for comparison. When the two are plotted, the final fit residuals are labeled as "Res 2 ". In these panels, the orange lines indicate the $3 \sigma$ limits. If the [O I] lines are not present, their restframe position is indicated in gray in both the spectrum and residual. Wavelengths are restframe.

Bottom. Line modeling for the Palomar data (same description as in previous panel) after stellar continuum modeling and subtraction (see Sect. 3.1 and Appendix A). The flux is in arbitrary units.
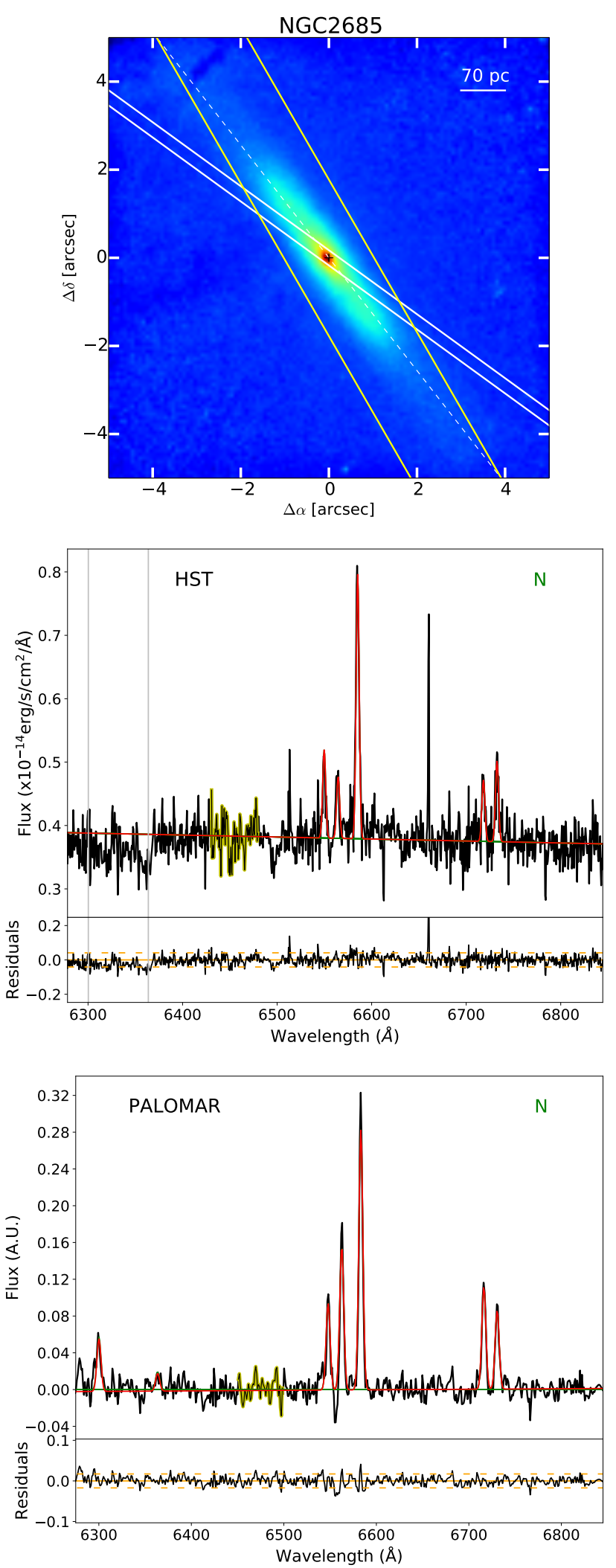

Fig. B.1. See text for a general description of the figure. NGC 2685: the $\mathrm{H} \alpha-[\mathrm{NII}]$ lines are unblended in both spectra. [O I] lines are not present in the HST/STIS spectrum, thus [S II] lines were used to model the other profiles. In the Palomar spectrum, [OI] lines are visible, however, no significant improvement was seen by using them to model the rest of the emission lines. All the lines are well-reproduced with a single narrow Gaussian component. 

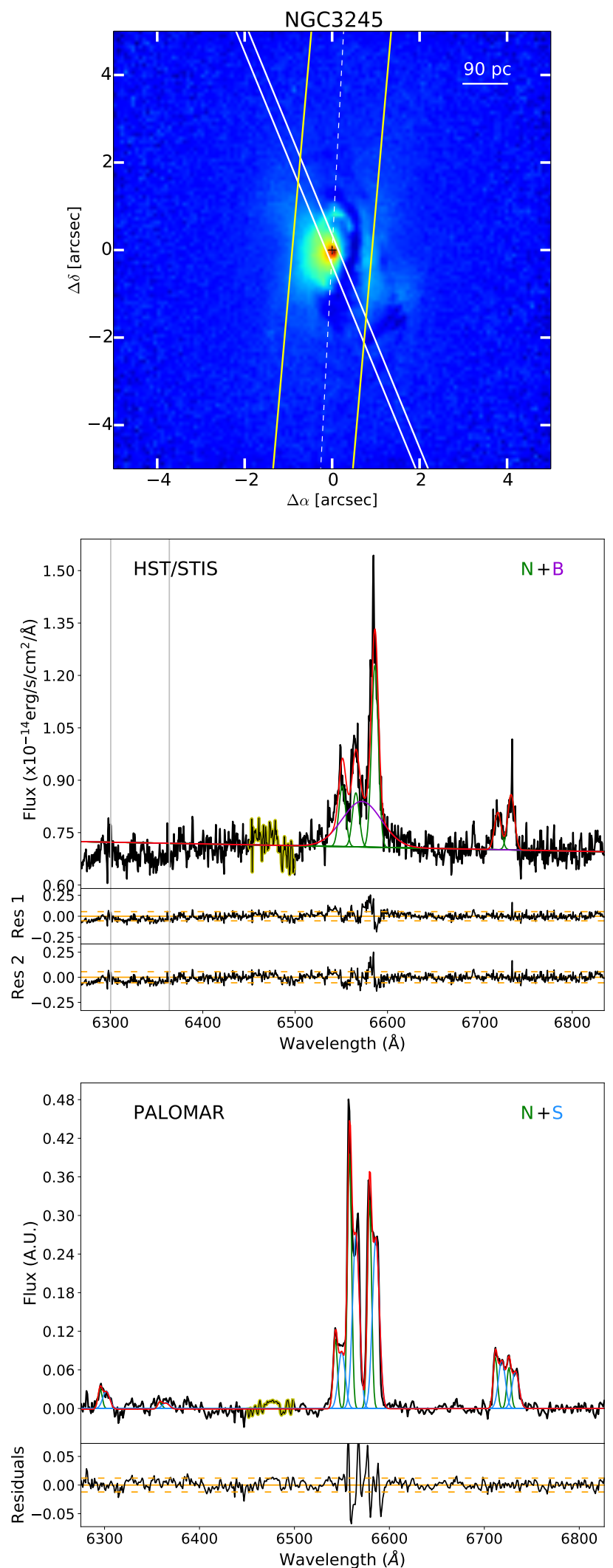

Fig. B.2. See text for a general description of the figure. NGC 3245 for HST/STIS spectra, emission lines are modeled with a single narrow Gaussian component. The residuals were improved by adding a broad component to $\mathrm{H} \alpha$. [O I] lines are not visible. In the Palomar spectrum, the double-peaked line profiles are modeled with two Gaussian components. As the [O I] line is rather faint, [S II] lines have been used for the modeling. $\mathrm{H} \alpha$ does not require any BLR-originated component.
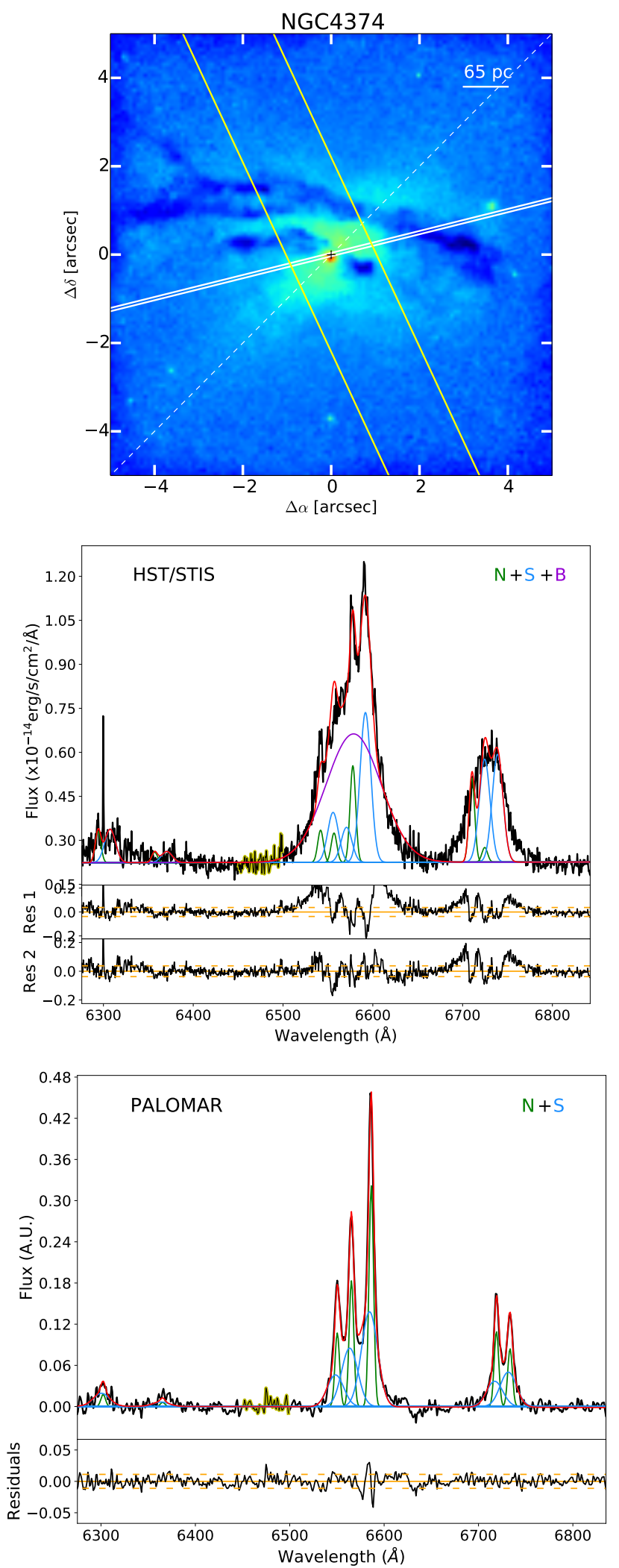

Fig. B.3. See text for a general description of the figure. NGC 4374: in the HST/STIS spectrum, [S II] lines are severely blended, and only the $[\mathrm{OI}] \lambda 6300 \AA$ line is visible (with low $\mathrm{S} / \mathrm{N}$ ). This complicated the modeling for the other lines, so the fit was performed considering the narrow peaks of [N II] $-\mathrm{H} \alpha$ blend (see Sect. 3), which results in high residuals under the $[\mathrm{S} \mathrm{II}]$ lines. A broad component is needed in this decomposition. For the Palomar spectrum, all the emission lines are well-modeled with two Gaussian components. 

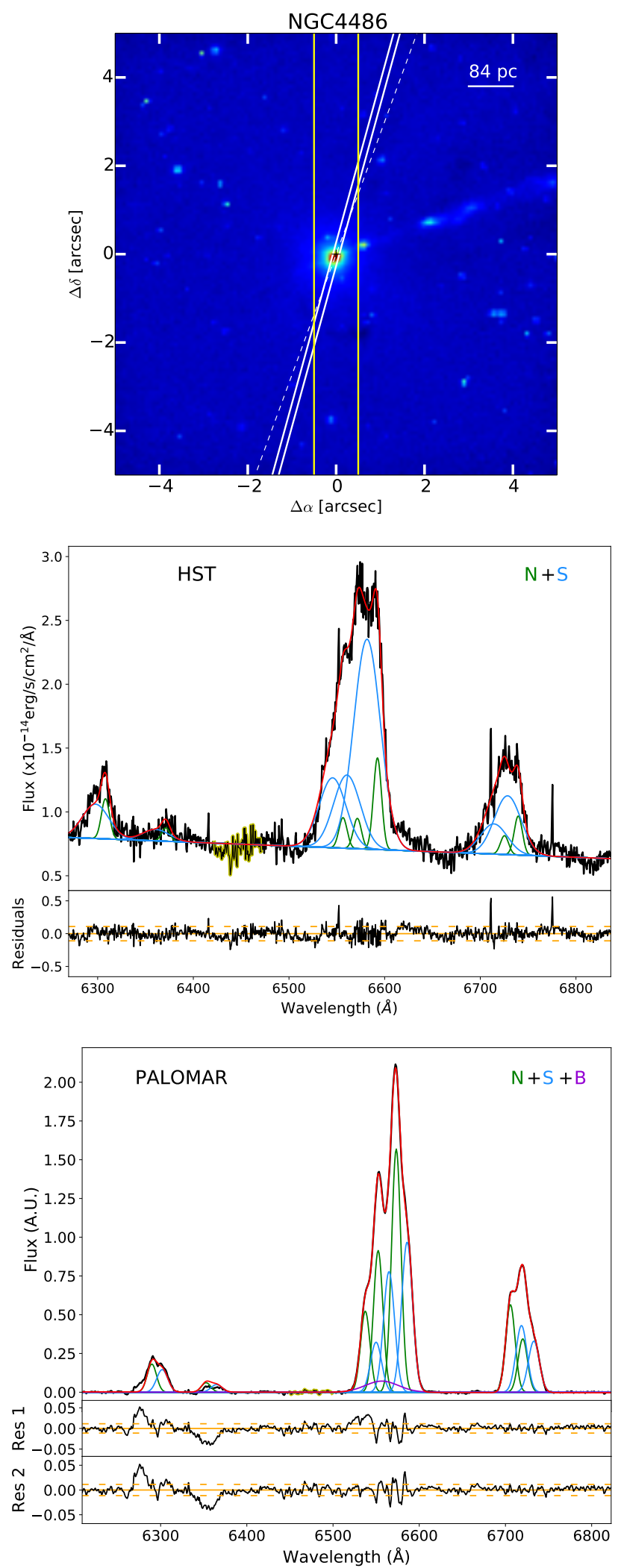

Fig. B.4. See text for a general description of the figure. NGC 4486 in the HST/STIS spectrum, two components are required to model the broad [OI] and [S II] emissions. No broad component is needed to model the $\mathrm{H} \alpha$ line profile. For the Palomar spectrum, two components have been used to reproduce the $[\mathrm{S} \mathrm{II}]$ and $[\mathrm{OI}]$ lines, but a very faint broad component is used for $\mathrm{H} \alpha$.
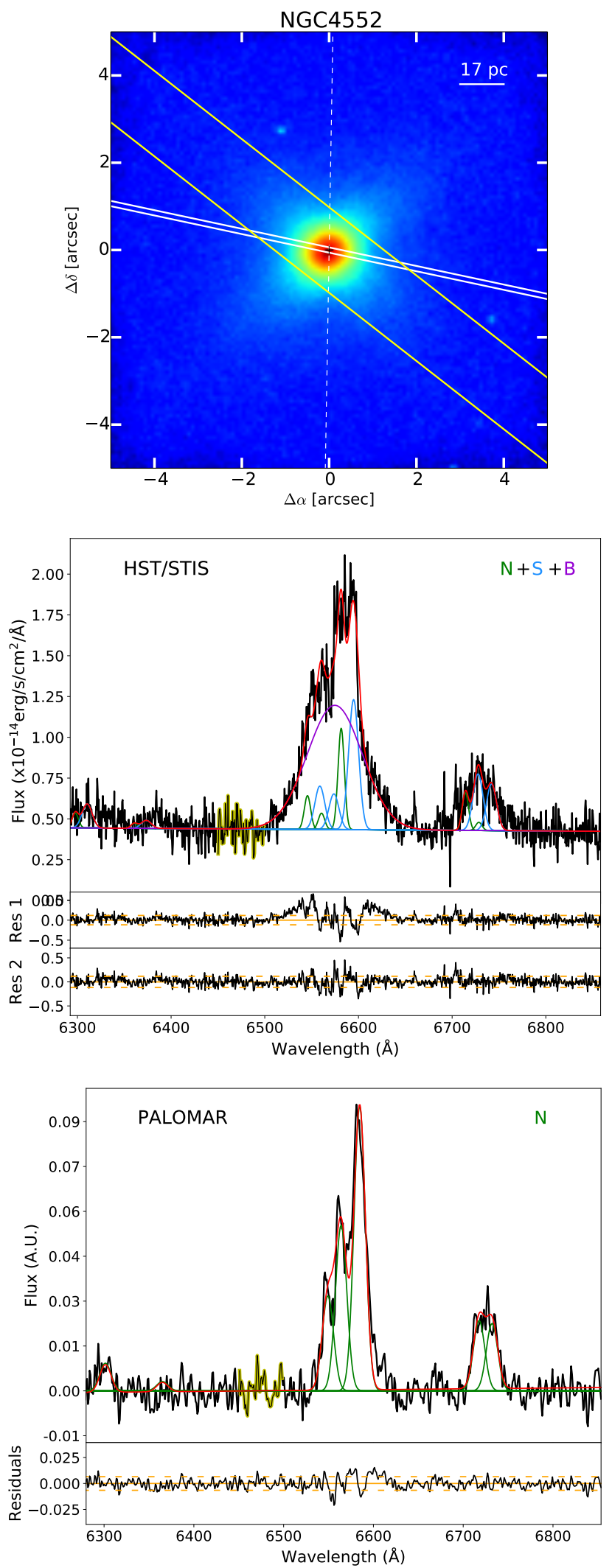

Fig. B.5. See text for a general description of the figure. NGC 4552: for HST/STIS spectrum, a second and a broad component were necessary to model emission lines. [O I] lines are very noisy and weak, thus they could not be used as a template for forbidden lines and narrow $\mathrm{H} \alpha$. A broad component is not required to fit the $\mathrm{H} \alpha$ profile. On the contrary, for the Palomar spectrum, all the emission lines can be reproduced with only a single component. 

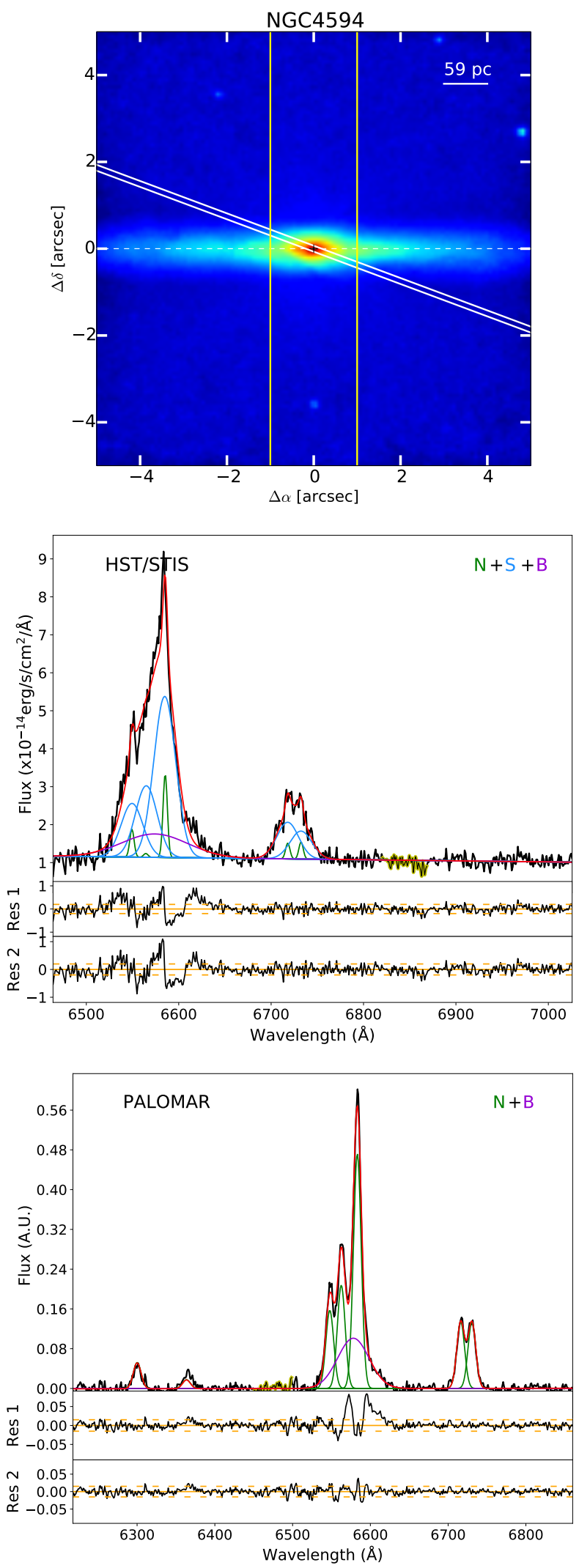

Fig. B.6. See text for a general description of the figure. NGC 4594: wavelength coverage of HST/STIS spectrum does not include [OI] lines. The [S II] lines have separate narrow profile peaks and a broader profile underneath. A broad component for $\mathrm{H} \alpha$ is to improve the fit. This broad component is also needed for the Palomar spectrum, although a single narrow Gaussian is sufficient for the forbidden lines.
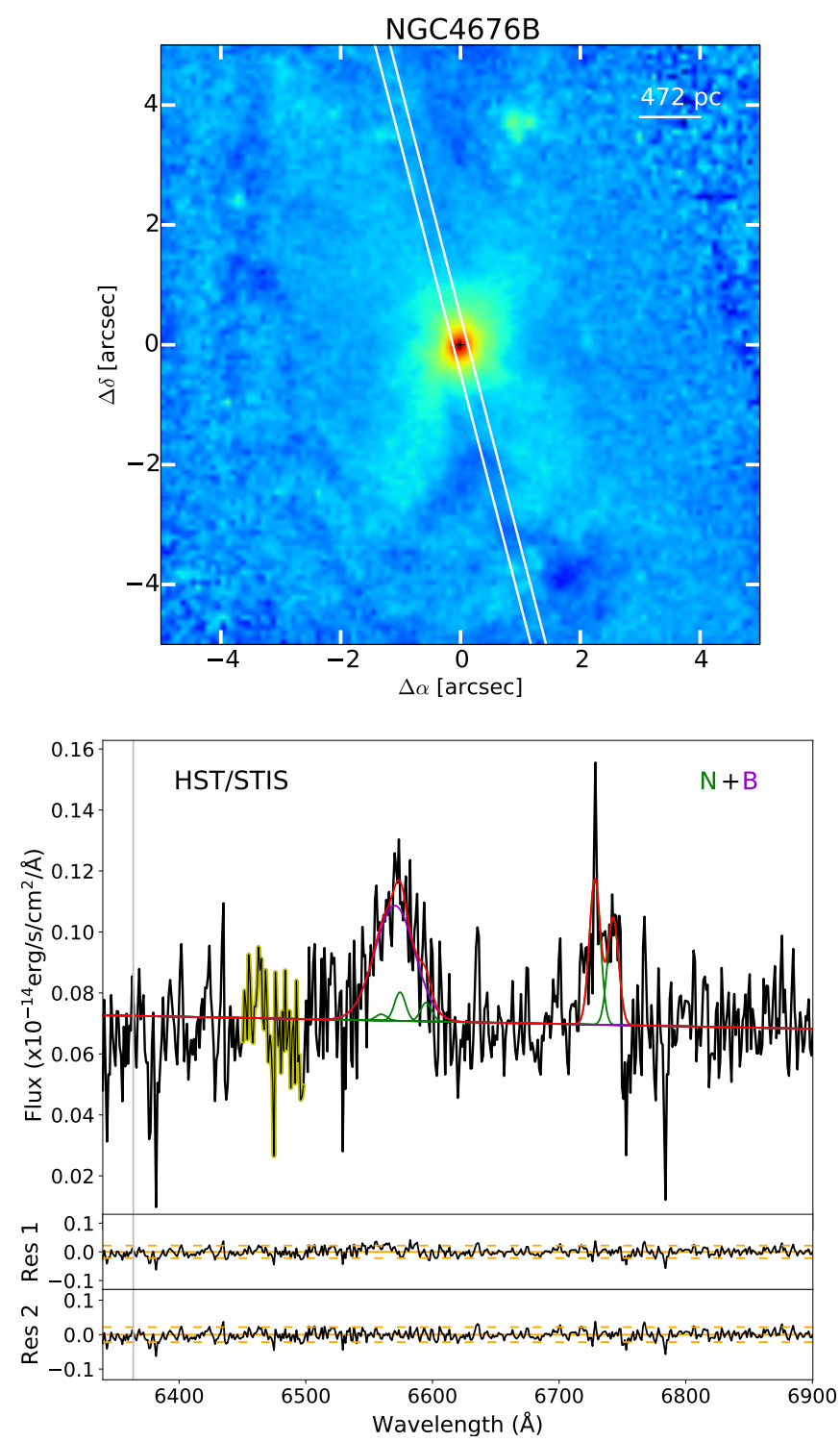

Fig. B.7. See text for a general description of the figure. NGC 4676B: $\mathrm{S} / \mathrm{N}$ of HST/STIS spectrum is generally low, and the quality of the fitting could be hampered. Both this and the wavelength range starting at $6340 \AA$ make the [OI] lines not visible, so only the S-method could be applied. The fit slightly improves by adding a broad component. There is no Palomar spectrum for this galaxy. The PA is not indicated, as this galaxy belongs to an interacting system, and there is no clear determination of it. 

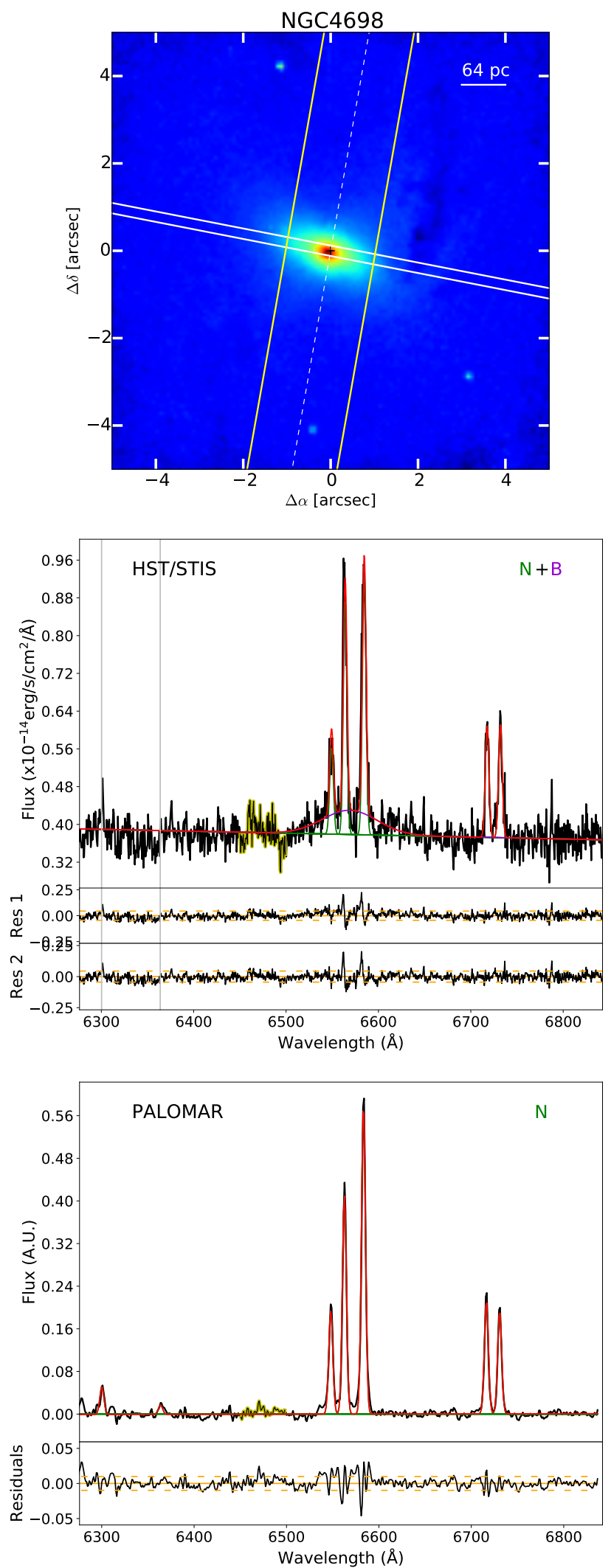

Fig. B.8. See text for a general description of the figure. NGC 4698: [OI] lines are not visible in HST/STIS spectrum. A narrow Gaussian component is sufficient to fit [S II] lines, and a broad component in $\mathrm{H} \alpha$ to improve the modeling, although its contribution to the global fit is rather weak. For the Palomar data a single narrow component is sufficient to model the lines.
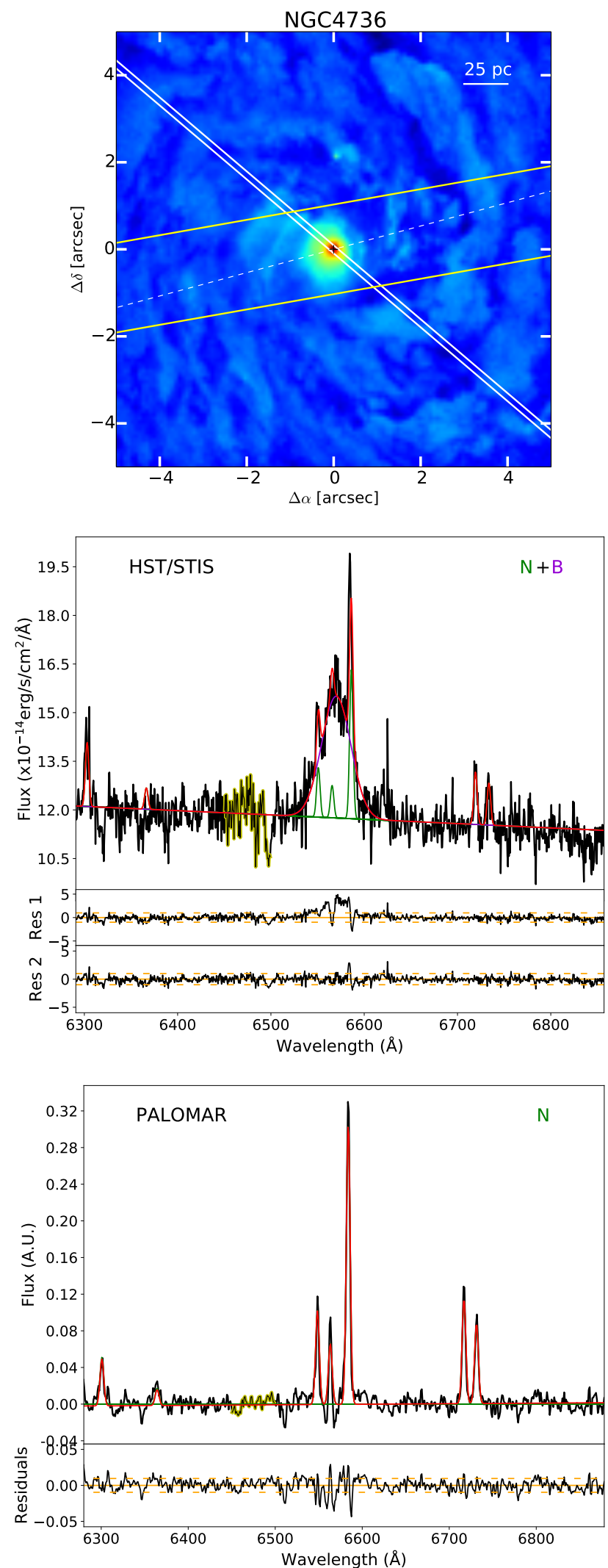

Fig. B.9. See text for a general description of the figure. NGC 4736: both $[\mathrm{S} \mathrm{II}]$ and $[\mathrm{OI}]$ lines are unblended in the HST/STIS spectrum and they clearly present narrow profiles. In the $\mathrm{H} \alpha-[\mathrm{N} \mathrm{II}]$ lines a broad component is visible, as already reported by Constantin et al. (2015). For the Palomar spectrum, a narrow component is sufficient for fitting all the emission lines considered in our study. 